\title{
Equilibria, Stability and Asymptotic Dominance in a Speculative Market with Heterogeneous Traders*
}

\author{
Mikhail Anufriev ${ }^{\mathrm{a}, \mathrm{b}, \uparrow} \quad$ Giulio Bottazzi $^{\mathrm{b}} \quad$ Francesca Pancotto $^{\mathrm{b}}$ \\ ${ }^{a}$ CeNDEF, Department of Economics, University of Amsterdam, \\ Roetersstraat 11, NL-1018 WB Amsterdam, Netherlands \\ ${ }^{\mathrm{b}}$ LEM, Sant'Anna School of Advanced Studies, \\ Piazza Martiri della Libertá 33, 56127 Pisa, Italy
}

15.09.2005

\begin{abstract}
We consider a simple pure exchange economy with two assets: one riskless, yielding a constant return on investment, and the other risky, paying a stochastic dividend. Trading takes place in discrete time and in each trading period the price of the risky asset is fixed by imposing a market clearing condition on the sum of individual demand functions. Individual demand for the risky asset is expressed as a fraction of wealth and depends on how traders forecast future price movement. Under these assumptions we derive the stochastic dynamical system describing the evolution of price and wealth.

We study the set of equilibria of this system for the case when arbitrarily many heterogeneous agents operate in the market, and we provide an asymptotic characterization of their relative performance. Abstracting from precise specification of agents' investment decisions, we show that all possible equilibrium returns belong to a one-dimensional "Equilibrium Market Line". It turns out that the system possesses isolated equilibria where a single agent dominates the market and continuous manifolds of equilibria where many agents hold finite wealth shares. The mechanism via which the market endogenously selects the dominant traders displays an optimal character in the neighborhood of the equilibria, but, at the same time, leads to the impossibility of defining a global dominance order relation among strategies.
\end{abstract}

JEL codes: G12, D83.

Keywords: Asset Pricing Model, Price and Wealth Dynamics, CRRA Framework, Equilibrium Market Line, Market Selection.

\footnotetext{
* Support by the Sant'Anna School of Advanced Studies (grant n. E6005GB) is gratefully acknowledged. We would like to thank Carl Chiarella, Alexander Coad, Cees Diks, Pietro Dindo, Cars Hommes, Florian Wagener, the participants of the 10th International Conference on Computing in Economics and Finance and three anonymous referees for helpful comments and insightful suggestions at various stages of this work. The usual disclaimers apply.

${ }^{\dagger}$ Corresponding author. Tel.: +31-20-5254248; fax: +31-20-5254349; e-mail: manoufriev@ sssup.it.
} 


\section{Introduction}

The standard classical models of financial markets, based on the presumption of the existence of a representative agent with full information and rational expectations, are regarded with an increasing skepticism by a growing number of scholars working in the field of finance and economic theory. In the last two decades, this dissatisfaction has led to the development of a new strand of literature built around quite heterogeneous contributions often joined under the label of "agent-based models". One could roughly divide these contributions into two partially overlapping classes. The first class contains models where results come from a strict analytical investigation. Among the examples surveyed in Hommes (2005) let us mention the models that bear a closer relationship with the present work: Chiarella (1992), Kirman (1993), Lux (1995), Brock and Hommes (1998), Gaunersdorfer (2000), Chiarella et al. (2002), Chiarella and He (2001, 2002b). The second class consists of models based on the presentation and discussion of extensive computer simulations. Such an approach was used, among many others, in Levy et al. (1994), Arthur et al. (1997), LeBaron et al. (1999), Lux and Marchesi (1999), Zschischang and Lux (2001), Farmer (2002) and Bottazzi et al. (2005). The reader is referred to LeBaron (2005) for a critical review of these works.

This paper is mainly intended as a contribution to the first class of models. We present a generic agent-based model and investigate its properties using analytical tools from the theory of dynamical systems. At the same time, our results shed new light on various regularities that have been previously, and repeatedly, observed in simulation-based studies.

We consider a simple, two-asset economy with a riskless security and an infinitely living risky equity. We model traders as adaptive heterogeneous agents who base their investment decisions on forecasts of future prices derived from the past market history. We assume that individual demand for the risky asset is expressed as a fraction of wealth. This assumption is consistent with, but not limited to, the behavior based on expected utility maximization with a constant relative risk aversion (CRRA) utility function.

The choice of a CRRA framework is somewhat unusual among agent-based analytical models, where the general preference seems to be for models in which the demand functions of agents are independent from the level of their wealth; a choice that is consistent with the maximization of a constant absolute risk aversion (CARA) expected utility. Examples of the latter approach are the Santa-Fe artificial market model described in Arthur et al. (1997) and LeBaron et al. (1999), and, among the analytical investigations, Brock and Hommes (1998) and its generalizations in Gaunersdorfer (2000), Chiarella and He (2002a) and Brock et al. (2005). The CARA framework is relatively more simple to handle, exactly because, in this case, the price dynamics is independent of the wealth distribution. This implies, as a direct economic consequence, that in these models all agents have the same impact on price formation, irrespective of their wealth. This relatively awkward property hints to the possibility that these models miss some important features of real markets. Moreover, there is empirical and experimental evidence (see the discussion in Levy et al. (2000)) suggesting that the behavior of traders and investors is rather consistent with a decreasing (with wealth) absolute risk aversion and/or with a constant relative risk aversion.

It is therefore not surprising that the CRRA framework has been the preferred choice both in the classical contributions, such as in Samuelson (1969), and in recent simulation-based models, which are not constrained by the need for mathematical simplicity, as in Levy et al. $(1994,1995)$ and Zschischang and Lux (2001). However, even with parsimoniously parameterized models there are few questions that can be definitely answered solely by use of computer simulations. Hence, an analytical investigation of such a framework seems necessary. To our knowledge only a few attempts in this direction have been carried out. These can be classified into two groups, according to the assumptions made about the underlying structure of the economy. The first group considers economies 
composed of short lived assets whose reward is generated by exogenously given processes; see for instance Blume and Easley (1992), Amir et al. (2005), Hens and Schenk-Hoppé (2005). The second group assumes infinitely lived securities whose investment returns possess an endogenously determined component (capital gain) that is the result of some intertemporal relationship between successive investment decisions; see Chiarella and He (2001, 2002b). This second class of security seems to more suitably describe the nature of the goods exchanged in many financial markets, like the global foreign exchange market, the bond market or the stock market.

The fact that it is impossible to derive a closed analytical expression for the solution of the CRRA expected utility maximization problem forced Chiarella and He to rely upon approximate expressions for the agent's demand function. Moreover, the requirement of keeping the dynamical system describing the evolution of the economy to a low dimension limited their investigation to the case in which only a small set of different strategies (one or two) operates at the same time in the market.

In the present paper we take a different approach and, while we keep exactly the same economic structure considered in Chiarella and $\mathrm{He}(2001,2002 \mathrm{~b})$ and a similar adaptive behavior for the agents, we extend the analysis in two directions. First, instead of deriving the individual demands from an (approximate) utility maximization principle, we model, in total generality, the agents' investment choices as smooth functions of the exponentially weighted moving average (EWMA) estimates of future asset return and variance. This investment function can be agent-specific, partially due to the fact that its shape should somehow depend on the agent's attitude towards risk, and partially due to the different possible ways in which an agent can transform an available information set (public or private) into predictions about the future. Second, we extend our analysis to the case in which a fixed, but arbitrarily large, number of agents operate in the market at the same time.

In this framework we are able to provide a complete characterization of the market equilibria and a description of their stability conditions in terms of a few parameters derived from the traders' investment functions. It turns out that, irrespective of the ecology of the agents operating in the market, the location of all steady-states can be illustrated by means of a simple function, the "Equilibrium Market Line" (EML). This separation between the underlying market structure which leads to the definition of the EML, and those aspects of the investment behaviors of traders, determining the precise equilibrium points, allows us to derive (notwithstanding the generality of the framework) several important conclusions. In particular, we find that, irrespective of the number of agents operating in the market and of the structure of their demand functions, only three types of equilibria are possible. First, generic equilibria associated with isolated fixed points, where a single agent asymptotically possesses the entire wealth of the economy. Second, generic "no-arbitrage" equilibria, where many agents coexist and both assets yield the same expected return. And, third, non-generic equilibria associated with continuous manifolds of fixed points, where many agents possess finite shares of the total wealth.

By means of stability analysis we are able to describe the relative asymptotic performances of the different investment functions and, ultimately, the mechanism via which the market selects the surviving traders. In this way the "quasi-optimal selection principle", originally formulated in Chiarella and He (2001) for (approximate) logarithmic utility maximizers, is extended to generic investment functions and arbitrarily large markets. This extension reveals the essentially local nature of the market selection process and the impossibility of defining any global dominance order relation among agents.

The rest of the paper is organized as follows. In the next Section the model is presented. We describe the structure of the economy, introduce the individual investment functions and derive the price and wealth dynamics as a multi-dimensional dynamical system. For ease of presentation, the discussion of the results is organized in successive steps, of increasing difficulty. In Section 3 we 
consider the simple case of homogeneous investment choices. We begin with the analysis of investment functions that depend on the EWMA estimator of the average return. The possible equilibria and their stability conditions are derived and briefly discussed. Then, we generalize our findings to the case in which the homogeneous investment function depends on the expected variance of future return. It turns out that accounting for the endogenous component of risk generated by the price volatility does not play any role in the determination of the equilibria and in their local stability. In Section 4 our analysis is extended to the general case of heterogeneous expectations, when many distinct agents operate in the market. We derive, under generic agents' preferences, the dynamical system describing the evolution of the economy, characterize all the possible equilibria and study their stability. In Section 5 we present two applications of the derived results. First, we consider a simple family of individual investment functions and discuss the effect of the different parameters on the location and stability of market equilibria. Second, we discuss, from a broader perspective, the mechanism by which a set of dominating behaviors emerges from the interaction of many heterogeneous agents. Section 6 summarizes our findings and lists some directions for further research. The proofs of all propositions are given in the Appendix.

\section{Model Structure}

We consider a simple pure exchange economy, populated by a fixed number $N$ of traders, where trading activities are supposed to take place in discrete time. The economy is composed of a riskless asset (bond) yielding in each period a constant interest rate $r_{f}>0$ and a risky asset (equity) paying a random dividend $D_{t}$ at the beginning of each period $t$. The riskless asset is considered the numéraire of the economy and its price is fixed to 1 . The ex-dividend price $P_{t}$ of the risky asset is determined at each period, on the basis of its aggregate demand, through a market-clearing condition.

With these specifications, let $x_{t, n}$ stand for the fraction of the wealth $W_{t, n}$ which, at time $t$, agent $n(n \in\{1, \ldots, N\})$ invests in the risky asset. After the trading session at time $t-1$, agent $n$ possesses $x_{t-1, n} W_{t-1, n} / P_{t-1}$ shares of the risky asset and $\left(1-x_{t-1, n}\right) W_{t-1, n}$ shares of the riskless asset. Agent $n$ then receives dividends payment $D_{t}$ per each risky asset and payment of riskless interest $r_{f}$ on the wealth invested in the riskless security. Therefore, before the trade at time $t$ the wealth of agent $n$, for any notional price $P$, reads

$$
W_{t, n}(P)=\left(1-x_{t-1, n}\right) W_{t-1, n}\left(1+r_{f}\right)+\frac{x_{t-1, n} W_{t-1, n}}{P_{t-1}}\left(P+D_{t}\right)
$$

and his individual demand for the risky asset becomes $x_{t, n} W_{t, n}(P) / P$. The actual price of the risky asset is fixed at the level for which aggregate demand is equal to aggregate supply. Assuming a constant supply of the risky asset, whose quantity can then be normalized to 1 , the price $P_{t}$ is defined as the solution of the equation

$$
\sum_{n=1}^{N} x_{t, n} W_{t, n}\left(P_{t}\right)=P_{t}
$$

The dynamics defined by equations (2.1) and (2.2) describe an exogenously growing economy. This may be seen by summing (2.1) over all agents, to obtain the dynamics of the total wealth $W_{t}$,

$$
W_{t}=W_{t-1}\left(1+r_{f}\right)+\left(P_{t}+D_{t}-P_{t-1}\left(1+r_{f}\right)\right)
$$

The first term in the right-hand side of (2.3) represents an "exogenous" expansion of the economy due to the continuous injection of new shares of the riskless asset, whose price remains unchanged 
under the assumption of totally elastic supply. The constant positive riskless return $r_{f}$ is responsible for this expansion. The second term represents the "excess" return on the risky asset. If one assumes that the market is perfectly efficient, so that the no-arbitrage hypothesis is valid, the expected value at time $t-1$ of this term has to be equal to zero, so that $E_{t-1}\left[W_{t}\right]=W_{t-1}\left(1+r_{f}\right)$. Consequently, the total wealth is characterized by an unbounded steady increase driven by the exogenous parameter $r_{f}$. It is convenient to remove this exogenous economic expansion from the dynamics of the model. To this end we introduce the rescaled variables

$$
w_{t, n}=\frac{W_{t, n}}{\left(1+r_{f}\right)^{t}} \quad, \quad p_{t}=\frac{P_{t}}{\left(1+r_{f}\right)^{t}} \quad, \quad e_{t}=\frac{D_{t}}{P_{t-1}\left(1+r_{f}\right)}
$$

The market intertemporal relations written in terms of these new variables read

$$
\begin{aligned}
& p_{t}=\sum_{n=1}^{N} x_{t, n} w_{t, n}, \\
& w_{t, n}=w_{t-1, n}+w_{t-1, n} x_{t-1, n}\left(\frac{p_{t}}{p_{t-1}}-1+e_{t}\right) \quad \forall n \in\{1, \ldots, N\} .
\end{aligned}
$$

The last equation shows that investment in the risky asset brings about the change in the agent's (rescaled) wealth through the dividend yield $e_{t}$ and also through the capital gain (loss) represented by the (rescaled) price return $r_{t}=p_{t} / p_{t-1}-1$. The relationship of $r_{t}$ with the return of unscaled prices $R_{t}$ can be obtained from (2.4) and reads

$$
R_{t}=\frac{P_{t}}{P_{t-1}}-1=r_{t}\left(1+r_{f}\right)+r_{f}
$$

Notice that a zero return for the rescaled prices $r_{t}=0$ corresponds to an unscaled return equal to the risk free interest rate $R_{t}=r_{f}$.

Equations (2.5) and (2.6) give the evolution of the state variables $w_{t, n}$ and $p_{t}$ over time, provided that the stochastic (due to random dividend payment $D_{t}$ ) yield process $\left\{e_{t}\right\}$ is given and the set of investment shares $\left\{x_{t, n}\right\}$ is specified. Concerning the former, the analysis of historical observations ${ }^{1}$ suggests that the yield is a bounded positive variable whose behavior is roughly stationary and independent of price. Thus we will require:

Assumption 1. The dividend yields $e_{t}$ are i.i.d. random variables obtained from a common distribution with positive support, mean value $\bar{e}$ and variance $\sigma_{e}^{2}$.

This assumption is common to a number of studies in the literature, e.g. Chiarella and He (2001), thus it allows us to maintain comparability with previous investigations. The final ingredient, the specification of agents' investment shares, is provided in the next Section.

\subsection{Investment functions and EWMA estimators}

In order to obtain a complete description of the evolution of the economy, one has to supplement the price and wealth dynamics described in (2.5) and (2.6) with the specification of the variables which describe the investment decisions of agents, $x_{t, n}$. We assume that these variables are idiosyncratic, endogenous and independent of the contemporaneous price and wealth levels. Important examples

\footnotetext{
${ }^{1}$ See, for instance, the data set on the Standard \& Poor's 500 index by Robert Shiller available online http://www.econ.yale.edu/ shiller/data.htm.
} 
of behaviors compatible with this specification include demand functions derived as a solution of an expected utility maximization problem with power utility function (implying CRRA behaviors). At the same time, this assumption rules out CARA-type behavior and also other possible dependencies in the determination of portfolio composition of agents, like an explicit relation of the present investment choice with the past investment choices or with the investment choices of other traders.

Furthermore, since we are mainly interested in the analysis of the effects of speculative behavior on the aggregate market performance, we leave aside those features which may occur under asymmetric knowledge of the underlying fundamental process. Thus, we assume that the structure of the yield process defined in Assumption 1 is known to everybody. Along the same lines, we assume that all agents base their investment decisions at time $t+1$ exclusively on the public information set $\mathcal{I}_{t}$ formed by past realized price returns, $\mathcal{I}_{t}=\left\{r_{t}, r_{t-1}, \ldots\right\}$. In this way past realizations of the yield process do not affect agents' decisions, which instead adapt to observed price fluctuations. One can refer to this investment behavior, common in the agent-based literature (e.g. Brock and Hommes (1998)), as "technical trading", stressing the similarity with trading practices observed in real markets.

Notwithstanding these restrictions, consideration of investment decisions based on the whole information set $\mathcal{I}_{t}$ would require, in a dynamic setting, to keep track of the entire past history of the market. In mathematical terms, this would lead to an infinite-dimensional dynamical system. To overcome this difficulty, and in line with the tradition of the agent-based literature, in the present paper we assume that the investment choice of each agent is obtained as a result of a two-step procedure $^{2}$ : in the first step the agent uses some set of estimators to form expectations about future price behavior starting from the information set $\mathcal{I}_{t}$; in the second step these expectations are plugged into an agent-specific choice function. If the estimators used in the first step admit a recursive definition, the description of the dynamics can be reduced to a low-dimensional system. In this paper we restrict our analysis to the case of exponentially weighted moving average (EWMA) estimators for the return and its variance. We introduce:

Assumption 2. For each agent $n$ there exists a parameter $\lambda_{n} \in[0,1)$ so that the agent's investment share can be obtained by means of a deterministic smooth investment function $f_{n}$ as

$$
x_{t+1, n}=f_{n}\left(y_{t, n}, z_{t, n}\right),
$$

where $y_{t, n}$ and $z_{t, n}$ are the expectations about future price return and variance obtained from information set $\mathcal{I}_{t}$ as follows

$$
\begin{aligned}
& y_{t, n}=\left(1-\lambda_{n}\right) \sum_{\tau=0}^{\infty} \lambda_{n}^{\tau} r_{t-\tau}, \\
& z_{t, n}=\left(1-\lambda_{n}\right) \sum_{\tau=0}^{\infty} \lambda_{n}^{\tau}\left(r_{t-\tau}-y_{t-\tau, n}\right)^{2} .
\end{aligned}
$$

The function $f_{n}$ on the right-hand side of (2.8) gives a complete description of the investment decision of agent $n$. The knowledge about the fundamental process, being complete and time invariant, is not explicitly inserted into the information set, rather it is considered embedded in the function $f_{n}$ itself, defined on the set $[-1,+\infty) \times[0,+\infty)$.

The decay factor $\lambda_{n}$ is a sort of "memory" parameter that determines how the relative weights in the averages (2.9) are distributed across more recent and older observations. The weights are declining geometrically into the past, so that the last available observation $r_{t}$ has the highest weight. The value $\lambda=0$ corresponds to the case of the naïve forecast, i.e. to that of the agent who uses

\footnotetext{
${ }^{2}$ The case with arbitrary investment functions mapping a finite but arbitrarily large past returns history into the future investment choice is considered in Anufriev and Bottazzi (2005).
} 
the last realized return as a predictor for the next period return. The use of the EWMA estimators seems reasonable in a dynamical setting where agents take into consideration the possibility that the "mood" prevailing in the market may change over time, so that more recent values of the price return could contain more information about future prices than the older ones ${ }^{3}$. The estimators (2.9) admit the recursive definition,

$$
\begin{aligned}
& y_{t, n}=\lambda_{n} y_{t-1, n}+\left(1-\lambda_{n}\right) r_{t} \\
& z_{t, n}=\lambda_{n} z_{t-1, n}+\lambda_{n}^{2}\left(1-\lambda_{n}\right)\left(r_{t}-y_{t-1, n}\right)^{2} .
\end{aligned}
$$

which will be used below in order to reduce the dimension of the dynamical system governing market evolution.

\subsection{The dynamical system for wealth shares and price return}

The dynamics described by (2.5) and (2.6) imply a simultaneous determination of the equilibrium price $p_{t}$ and of the agents' wealth $w_{t, n}$. Due to this simultaneity, the $N+1$ equations in (2.5) and (2.6) define the state of the system at time $t$ only implicitly ${ }^{4}$.

The transformation of the implicit dynamics into economically meaningful explicit dynamics, i.e. dynamics where the asset prices remain positive, is not generally possible and entails further constraint on the set of market positions allowed to agents. The next Proposition derives these constraints and provides an explicit description of the dynamical system defined in (2.5) and (2.6). Before that, let us introduce some notation that will prove useful in formulating the market dynamics in a more compact form. Let $a_{n}$ be an agent-specific variable, possibly dependent on time $t$. We denote with $\langle a\rangle_{t}$ its wealth weighted average, on the population of agents, at time $t$, i.e.

$$
\langle a\rangle_{t}=\frac{\sum_{n=1}^{N} a_{n} w_{t, n}}{w_{t}}=\sum_{n=1}^{N} a_{n} \varphi_{t, n} \quad, \quad \text { where } \quad w_{t}=\sum_{n=1}^{N} w_{t, n}
$$

represents the total wealth in the economy and $\varphi_{t, n}=w_{t, n} / w_{t}$ is the $n$-th agent's wealth share.

Proposition 2.1. Let us assume that initial price $p_{0}$ is positive. Under Assumption 2, from equations (2.5) and (2.6) it is possible to derive a map $\mathbb{R}_{+}^{N} \rightarrow \mathbb{R}_{+}^{N}$ that describes the evolution of wealth $w_{t, n} \forall n \in\{1, \ldots, N\}$ with positive prices $p_{t} \in \mathbb{R}_{+} \forall t>0$ provided that

$$
\left(\left\langle x_{t}\right\rangle_{t}-\left\langle x_{t} x_{t+1}\right\rangle_{t}\right)\left(\left\langle x_{t+1}\right\rangle_{t}-\left(1-e_{t+1}\right)\left\langle x_{t} x_{t+1}\right\rangle_{t}\right)>0 \quad \forall t
$$

If this is the case, the growth rate of the (rescaled) price $r_{t+1}=p_{t+1} / p_{t}-1$ reads

$$
r_{t+1}=\frac{\left\langle x_{t+1}-x_{t}\right\rangle_{t}+e_{t+1}\left\langle x_{t} x_{t+1}\right\rangle_{t}}{\left\langle x_{t}\left(1-x_{t+1}\right)\right\rangle_{t}}
$$

the individual growth rates of the (rescaled) wealth $\rho_{t+1, n}=w_{t+1, n} / w_{t, n}-1$ are given by

$$
\rho_{t+1, n}=x_{t, n}\left(r_{t+1}+e_{t+1}\right) \quad \forall n \in\{1, \ldots, N\} \quad,
$$

\footnotetext{
${ }^{3}$ The EWMA estimators are widely applied by real market participants in their forecasting activity, see e.g. the technical document of the RiskMetrics Group ${ }^{\mathrm{TM}}$ (J.P.Morgan, 1996), and were also employed in the theoretical models of Gaunersdorfer (2000) and Bottazzi (2002).

${ }^{4}$ The $N$ variables $w_{t, n}$ defined in (2.6) appear on the right-hand side of (2.5), and, at the same time, the variable $p_{t}$ defined in (2.5) appears on the right-hand side of (2.6).
} 
and the agents' (rescaled) wealth shares $\varphi_{t, n}$ evolve accordingly to

$$
\varphi_{t+1, n}=\varphi_{t, n} \frac{1+\left(r_{t+1}+e_{t+1}\right) x_{t, n}}{1+\left(r_{t+1}+e_{t+1}\right)\left\langle x_{t}\right\rangle_{t}} \quad \forall n \in\{1, \ldots, N\} .
$$

Proof. See appendix A.

The market evolution is explicitly described by the system of $N+1$ equations in (2.13) and (2.14), or, equivalently, in (2.13) and (2.15). The price dynamics can be derived from (2.13) in a straight-forward manner, but the price will stay positive only as long as two requirements are met. First, the initial price $p_{0}$ is positive and, second, the inequality in (2.12) is fulfilled at each time step. It may be quite cumbersome to analytically investigate the latter condition in total generality. From a practical point of view, it is better to make this condition stronger and, at the same time, to simplify it. A possibility is provided by the following

Proposition 2.2. Consider the system defined in Proposition 2.1. If there exist two real values $x_{\min }$ and $x_{\max }$ such that

$$
0<x_{\min } \leq x_{t, n} \leq x_{\max }<1 \quad \text { and } \quad e_{t} \geq 0, \quad \forall t, \forall n \in\{1, \ldots, N\} \quad,
$$

then condition (2.12) is always satisfied and the dynamics of (2.13) and (2.15) are bounded, that is there exist constants $r_{\min }, r_{\max }, \rho_{\min }$ and $\rho_{\max }$, such that

$$
r_{\min } \leq r_{t} \leq r_{\max } \quad \text { and } \quad \rho_{\min } \leq \rho_{t, n} \leq \rho_{\max } \quad \forall t, \forall n \in\{1, \ldots, N\} \quad
$$

Proof. See appendix B.

Thus, if all possible investment choices are confined on some compact subinterval in $(0,1)$, then equations (2.13) and (2.15) give well-defined dynamics in terms of price and wealth shares.

The issue of the positiveness of the initial price deserves a brief discussion. Notice that this assumption is often not binding, since it can be satisfied for (almost) any set of initial investment shares by means of an appropriate choice of the initial wealth, which is not restricted to be positive. Let us consider, for example, the case of a market with a single agent possessing an initial endowment of $B_{0}$ bonds and 1 equity (i.e. the total supply of equities). If $x_{0}$ is the share of wealth the agent decides to invest in the risky security, the price is the solution of the equation

$$
x_{0}\left(B_{0}+P_{0}\right)=P_{0} \text {. }
$$

The left- and the right-hand sides of this equation are linear functions of $P_{0}$ and the equilibrium price is defined as the intersection of these two straight lines. One immediately sees that if $x_{0} \in(0,1)$, the price is positive when $B_{0}>0$. On the other hand, for either $x_{0}>1$ or $x_{0}<0$, the price is positive when $B_{0}<0$, i.e. when the agent has a "debt" in the riskless security. Finally, if the investment share is 0 or 1 , a positive price cannot be fixed.

Summarizing, in this Section we have derived the evolution of price and wealth in a market defined by Assumption 1 and populated by agents behaving in accordance with Assumption 2. This framework covers a considerable extent of possible behavioral specifications. While the set of available estimators used by agents is fixed, we allow agents to have heterogeneous expectations (different values of the parameter $\lambda$ ) and different and completely arbitrary investment functions $f_{n}$. At the same time, our market structure is defined in such a way that the dynamics in (2.13) and (2.15) do not depend on the price level directly, but, instead, are defined in terms of price return and dividend yield. Since in these equations only the wealth ratios defined in (2.11) appear, and these are insensitive to a homogeneous rescaling of the wealth levels, the equilibria of the model can be identified as states of steady expansion (or contraction) of the economy. 


\section{The Economy with Homogeneous Agents}

In this Section we perform the equilibrium and stability analysis of the dynamics derived above for the simplest situation in which traders possess homogeneous beliefs and preferences, in other words, share the same investment function. It is clear from (2.1) that, in this case, the dynamics of the economy are equivalent to those obtained when a single agent operates in the market, i.e. when $N=1$. We consider this "single agent" case at length, due to its relevance for the heterogeneous agent case discussed later.

We start with the analysis of a single agent whose investment function ${ }^{5} f$ only depends on estimated future price return $y$. Subsequently, we extend the analysis to an investment function which also depends on the estimated variance $z$, as in (2.8). Splitting the investigation into two steps helps in understanding the different effects that the dependence on $y$ and $z$ has on the market dynamics. Before starting our analysis, it is useful to introduce the following

Definition 3.1. The Equilibrium Market Line (EML) is the function $l(r)$ defined according to

$$
l(r)=\frac{r}{\bar{e}+r},
$$

where $\bar{e}$ stands for the mean yield value as defined in Assumption 1.

\subsection{Investment based on forecasted return}

In the single agent case the dynamical system describing the market evolution can be simplified since the explicit evolution of the wealth shares in (2.15) is not needed. Substituting (2.13) into the first equation in (2.10) we reduce the market evolution to a two-dimensional stochastic system

$$
\left\{\begin{array}{l}
x_{t+1}=f\left(y_{t}\right), \\
y_{t+1}=\lambda y_{t}+(1-\lambda) \frac{f\left(y_{t}\right)-x_{t}+e_{t+1} x_{t} f\left(y_{t}\right)}{x_{t}\left(1-f\left(y_{t}\right)\right)} .
\end{array}\right.
$$

The dynamics of the price return (2.13) is responsible for the fraction on the right-hand side of the second equation. The stochastic nature of (3.2) originates from the random dividend yield $\left\{e_{t}\right\}$. In order to study the asymptotic properties of the system, we replace the realizations of the yield process by its mean value $\bar{e}$ and consider the fixed points of the resulting deterministic skeleton. The next result characterizes the existence and location of these fixed points ${ }^{6}$.

Proposition 3.1. Let $\left(x^{*}, y^{*}\right)$ be a fixed point of the deterministic skeleton of system (3.2). Then:

(i) The equilibrium return $r^{*}$ and the equilibrium investment share $x^{*}$ satisfy

$$
l\left(r^{*}\right)=f\left(r^{*}\right), \quad x^{*}=f\left(r^{*}\right),
$$

and the equilibrium value of the predictor coincides with the equilibrium price return, $y^{*}=r^{*}$.

(ii) The equilibrium is feasible, i.e. the prices are positive, if either $x^{*}<1$ or $x^{*}>1 /(1-\bar{e})$.

(iii) The equilibrium growth rate of agent's wealth is equal to price return, $\rho^{*}=r^{*}$.

\footnotetext{
${ }^{5}$ Since only one agent is present in the market, we omit the index 1 from any agent-specific variable.

${ }^{6}$ All following results are valid under the condition that $\bar{e}<1$ which seems robustly satisfied by real data.
} 
Proof. See appendix C.

According to Proposition 3.1(i) the equilibrium price return $r^{*}$ coincides with the prediction of the EWMA estimator $y^{*}$. This is an important consistency result which must hold for any meaningful economic dynamics. Equations (3.3) provide a simple geometric characterization of all possible equilibria: they can be obtained as the intersections of the investment function $f$ with the Equilibrium Market Line $l$ defined in (3.1). Notice that the EML is made of two branches separated by a vertical asymptote at $-\bar{e}$. Thus, for $r^{*}=-\bar{e}$ no equilibria exist. Indeed, if the positive dividend yield were exactly offset by a negative price return, the (rescaled) wealth of the agent would be constant over time and the investment share would increase, unboundedly, at a rate $1-\bar{e}$. Proposition 3.1(ii) shows that not all equilibria are economically meaningful, though. Provided that initial price is positive, the equilibrium return generates positive prices only in those equilibria where $r^{*}>-1$, or, equivalently, where $x^{*}$ belongs to the intervals $(-\infty, 1)$ or $(1 /(1-\bar{e}),+\infty)$. This condition is, indeed, the equilibrium version of inequality (2.12). Finally, Proposition 3.1(iii) states that the growth rate of the agent's wealth coincides with the price return in the equilibrium.

In the left panel of Fig. 1 two investment functions (thick lines) are reported. The equilibria are identified as the intersections of these curves with the EML (thin line). The abscissa of the intersection gives the value of the equilibrium return $r^{*}$ while the ordinate gives the equilibrium investment share $x^{*}$. One can distinguish between three qualitatively different scenarios.

For those equilibria with $r^{*} \in[-1,-\bar{e})$ the investment in the risky asset is characterized by negative gross return $\left(r^{*}+\bar{e}<0\right)$ and the agent constantly borrows resources to invest in the risky asset $\left(x^{*}>1\right)$. In these equilibria the total wealth grows at a negative rate ${ }^{7}$ and, according to the example on page 8 , the agent possesses a negative amount of the riskless security.

If $r^{*} \in(-\bar{e}, 0)$, the capital gain on the risky asset is negative, nevertheless the gross return is positive due to the dividend yield. Agent invests a negative share of wealth in the risky asset $\left(x^{*}<0\right)$ but the total wealth is also negative to guarantee positiveness of prices (cf. (2.5)) and grows at a negative rate. This is the case of equilibrium $S_{2}$ of the linear investment function in the left panel of Fig. 1.

Finally, if $r^{*} \in(0,+\infty)$, the price return is positive, a positive fraction of wealth is invested in the risky asset $\left(x^{*} \in(0,1)\right)$ and the agent's wealth return is positive. This is the case of equilibrium $U_{2}$ of the linear investment function and equilibria $S_{1}$ and $U_{1}$ of the nonlinear function in the left panel of Fig. 1.

While the equilibria do not depend on the agent's forecast parameter $\lambda$, this parameter is important in deciding their stability, as we show in the following

Proposition 3.2. The fixed point $\left(x^{*}, y^{*}\right)$ of the deterministic skeleton of system (3.2) is (locally) asymptotically stable if

$$
\frac{f^{\prime}\left(r^{*}\right)}{l^{\prime}\left(r^{*}\right)} \frac{1}{r^{*}}<\frac{1}{1-\lambda}, \quad \frac{f^{\prime}\left(r^{*}\right)}{l^{\prime}\left(r^{*}\right)}<1 \quad \text { and } \quad \frac{f^{\prime}\left(r^{*}\right)}{l^{\prime}\left(r^{*}\right)} \frac{2+r^{*}}{r^{*}}>-\frac{1+\lambda}{1-\lambda}
$$

where $f^{\prime}\left(r^{*}\right)$ and $l^{\prime}\left(r^{*}\right)$ stand for the first derivative of the investment function $f(y)$ and of the EML $l(r)$ computed in equilibrium, respectively.

The equilibrium is unstable if at least one of the inequalities in (3.4) holds with the opposite (strict) sign. The stability is lost through a Neimark-Sacker, fold or flip bifurcation if the first, the second or the third inequality in (3.4), respectively, is violated.

Proof. See appendix D. 

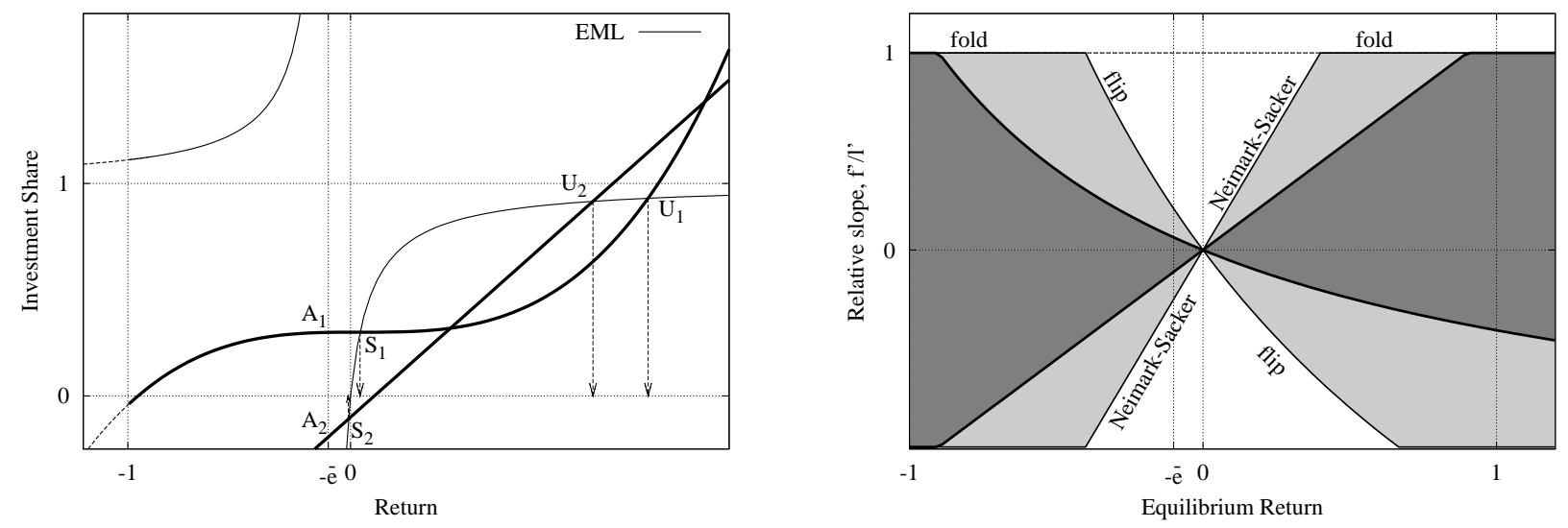

Figure 1: Equilibria and stability for the single agent system. Left panel: the equilibria for two different investment functions (thick lines) defined as the intersection with the EML (thin line). Right panel: equilibrium stability region for two different values of $\lambda$ in coordinates $r^{*}$ and $f^{\prime}\left(r^{*}\right) / l^{\prime}\left(r^{*}\right)$. For $\lambda=0.1$, the fixed point is locally stable if $\left(r^{*}, f^{\prime} / l^{\prime}\right)$ belongs to the dark-gray region. When the value of $\lambda$ increases to 0.6 , the stability region expands and becomes the union of the dark-gray and light-gray areas.

This Proposition highlights the role of the parameters $\lambda, r^{*}$ and the relative slope of the investment function w.r.t. the EML, $f^{\prime}\left(r^{*}\right) / l^{\prime}\left(r^{*}\right)$, for the local stability of the equilibria. The stability regions in terms of $r^{*}$ and $f^{\prime}\left(r^{*}\right) / l^{\prime}\left(r^{*}\right)$ for two different values of $\lambda$ are shown in the right panel of Fig. 1 as differently shaded gray areas. The stability region increases together with the value of $\lambda$. This parameter plays an important role in the stability of the system. Indeed, since the value of $\lambda$ does not affect the position of the fixed point, any equilibrium with $f^{\prime} / l^{\prime}<1$ and $r^{*} \neq 0$ becomes stable when $\lambda$ takes a sufficiently large value.

The right panel of Fig. 1 also shows the types of bifurcation exhibited when one of the stability conditions in (3.4) is violated. For relatively small absolute values of the equilibrium return the system can lose stability through either a flip or a Neimark-Sacker bifurcation. In this case, if the system is perturbed away from equilibrium, for example, by a small shock to the investment share, the large absolute value of $f^{\prime}$ is responsible for an amplification of this perturbation, based on the feedback mechanism linking investment share and market return, which ultimately leads to oscillatory behavior of the system. For larger absolute values of the equilibrium return, if the investment function is steeper at the equilibrium than the EML, the equilibrium is lost through a fold bifurcation, which implies local exponential growth of the price returns. Notice that equilibria $U_{1}$ and $U_{2}$ in Fig. 1 (left panel) can be immediately recognized as unstable, since they clearly violate the second inequality in (3.4).

\subsection{Investment based on forecasted return and variance}

Let us now move to the case of a single agent whose investment function depends on both estimators defined on page 6. One can see this as an extension of the previous analysis to the case of an agent who takes investment decisions based on both expected profit and the endogenous component of risk. Indeed, notice that (2.14) explicitly identifies two different risks components in the profit coming from investment in the risky asset: the stochastic yield process $\left\{e_{t}\right\}$ and the possibly volatile

\footnotetext{
${ }^{7}$ Remember that the analysis is performed with respect to the rescaled variables as defined in (2.4). Negative return corresponds to the return less than $r_{f}$ in terms of the unscaled variables.
} 
return dynamics $\left\{r_{t}\right\}$. The former is assumed exogenous (Assumption 1) and perfectly known to agent (Assumption 2). The latter is, on the contrary, endogenous and not perfectly known. The EWMA estimator $z_{t}$ introduced in (2.9) can be thought of as a measure of this second component of risk. Combining (2.8), (2.10) and (2.13), one gets the following three-dimensional system:

$$
\left\{\begin{array}{l}
x_{t+1}=f\left(y_{t}, z_{t}\right) \\
y_{t+1}=\lambda y_{t}+(1-\lambda) \frac{f\left(y_{t}, z_{t}\right)-x_{t}+e_{t+1} x_{t} f\left(y_{t}, z_{t}\right)}{x_{t}\left(1-f\left(y_{t}, z_{t}\right)\right)} \\
z_{t+1}=\lambda z_{t}+\lambda^{2}(1-\lambda)\left[\frac{f\left(y_{t}, z_{t}\right)-x_{t}+e_{t+1} x_{t} f\left(y_{t}, z_{t}\right)}{x_{t}\left(1-f\left(y_{t}, z_{t}\right)\right)}-y_{t}\right]^{2}
\end{array}\right.
$$

The following result implies that the EML can still be used for the characterization of equilibria.

Proposition 3.3. Let $\left(x^{*}, y^{*}, z^{*}\right)$ be a fixed point of the deterministic skeleton of system (3.5) and let $r^{*}$ denote the price return at this point. Then:

(i) The equilibrium return $r^{*}$ and the equilibrium investment share $x^{*}$ satisfy

$$
l\left(r^{*}\right)=f\left(r^{*}, 0\right), \quad x^{*}=f\left(r^{*}, 0\right) .
$$

The equilibrium value of the return estimator coincides with the equilibrium price return, $y^{*}=r^{*}$, while the equilibrium value of the variance estimator is zero, $z^{*}=0$.

(ii) The equilibrium is feasible, if either $x^{*}<1$ or $x^{*}>1 /(1-\bar{e})$.

(iii) The equilibrium growth rate of the agent's wealth is equal to price return, $\rho^{*}=r^{*}$.

All the differences between this statement and Proposition 3.1 are in the first item. The consistency result for the equilibrium return estimator is confirmed and extended to the variance estimator $z^{*}$, whose value at equilibrium becomes zero, as expected for a constant return. The conditions characterizing the equilibrium investment share and return have slightly changed, because the investment function $f$ now depends on two variables. However, if one considers the restriction of $f$ to the set $z=0$, the former characterization of the equilibria as the intersections with the EML is still valid.

The following result provides the conditions for the stability of the equilibria.

Proposition 3.4. The fixed point $\left(x^{*}, y^{*}, z^{*}\right)$ of the deterministic skeleton of system (3.5) is (locally) asymptotically stable if

$$
\frac{f_{y}^{\prime}\left(r^{*}, 0\right)}{l^{\prime}\left(r^{*}\right)} \frac{1}{r^{*}}<\frac{1}{1-\lambda}, \quad \frac{f_{y}^{\prime}\left(r^{*}, 0\right)}{l^{\prime}\left(r^{*}\right)}<1 \quad \text { and } \quad \frac{f_{y}^{\prime}\left(r^{*}, 0\right)}{l^{\prime}\left(r^{*}\right)} \frac{2+r^{*}}{r^{*}}>-\frac{1+\lambda}{1-\lambda}
$$

where $f_{y}^{\prime}$ is, in usual notation, the partial derivative of the investment function with respect to the first variable $y$ and $l^{\prime}$ is the first derivative of the EML.

The equilibrium is unstable if at least one of the inequalities in (3.7) holds with the opposite (strict) sign. The system exhibits a Neimark-Sacker, fold or flip bifurcation if the first, the second or the third inequality in (3.4), respectively, becomes an equality.

Proof. See appendix E. 
Since $f_{y}^{\prime}\left(r^{*}, 0\right)$ is exactly the derivative of the restriction of $f$ to the set $z=0$, it is clear that this restriction reduces this last Proposition to Proposition 3.2.

The most interesting result of Proposition 3.4 is that the introduction of a measure of endogenous risk into the agent's investment function, albeit changing (in general) the global behavior of the system, does not have any qualitative impact on the local dynamics in the neighborhood of an equilibrium $^{8}$. This is not to say that investor's attitude towards risk is irrelevant for the stability of the system. Risk aversion is a property of the specific functional form of the investment function, something that cannot be investigated here. The point of the last two Propositions is that for any investment function $f(y, z)$ there exists an "equivalent" function depending solely on the estimator $y$, namely $f(y, 0)$, that provides all the information concerning the allowed equilibria and their local stability. As an example in Section 5.1 will demonstrate, if a specific investment function contains a risk aversion parameter, this parameter will in general affect its restriction to the $z=0$ plane.

\section{The Economy with Heterogeneous Agents}

In this Section we consider the case in which many heterogeneous agents, with different investment functions, operate in the market. All these functions depend on the EWMA estimators of future price dynamics, but we allow the parameters of these estimators to differ among agents.

\subsection{The dynamical system}

The main difference of the heterogeneous setting with respect to the single agent case concerns the role of the wealth dynamics. Indeed, the evolution of wealth shares is no longer decoupled from the dynamics of price and, consequently, both (2.13) and the entire set of equations in (2.15) become relevant. Under the conditions in Assumption 2 the evolution of the economy can be described in terms of the variables $x_{t, n}, y_{t, n}$ and $z_{t, n}$ for $n \in\{1, \ldots, N\}$ and of $\varphi_{t, n}$ for $n \in\{1, \ldots, N-1\}$ as in the following

Lemma 4.1. The dynamics defined by (2.13) and (2.15) with investment choices (2.8) can be described by means of the following system of $4 N-1$ first-order difference equations

$$
\begin{aligned}
& \mathcal{X}:\left[\begin{array}{rll}
x_{t+1,1} & = & f_{1}\left(y_{t, 1}, z_{t, 1}\right) \\
\vdots & \vdots & \vdots \\
x_{t+1, N} & = & f_{N}\left(y_{t, N}, z_{t, N}\right)
\end{array}\right. \\
& \mathcal{Y}:\left[\begin{array}{rll}
y_{t+1,1} & = & \lambda_{1} y_{t, 1}+\left(1-\lambda_{1}\right) r_{t+1} \\
\vdots & \vdots & \vdots \\
y_{t+1, N} & = & \lambda_{N} y_{t, N}+\left(1-\lambda_{N}\right) r_{t+1}
\end{array}\right. \\
& \mathcal{Z}:\left[\begin{array}{rll}
z_{t+1,1} & = & \lambda_{1} z_{t, 1}+\lambda_{1}^{2}\left(1-\lambda_{1}\right)\left(r_{t+1}-y_{t, 1}\right)^{2} \\
\vdots & \vdots & \vdots \\
z_{t+1, N} & = & \lambda_{N} z_{t, N}+\lambda_{N}^{2}\left(1-\lambda_{N}\right)\left(r_{t+1}-z_{t, N}\right)^{2}
\end{array}\right. \\
& \mathcal{W}:\left[\begin{array}{ccc}
\varphi_{t+1,1} & =\Phi_{1}\left(x_{t, 1}, \ldots, x_{t, N} ; \varphi_{t, 1}, \ldots, \varphi_{t, N-1} ; e_{t+1} ; r_{t+1}\right) \\
\vdots & \vdots & \vdots \\
\varphi_{t+1, N-1} & = & \Phi_{N-1}\left(x_{t, 1}, \ldots, x_{t, N} ; \varphi_{t, 1}, \ldots, \varphi_{t, N-1} ; e_{t+1} ; r_{t+1}\right)
\end{array}\right.
\end{aligned}
$$

\footnotetext{
${ }^{8}$ This result is in line with Gaunersdorfer (2000) who shows, in a model with CARA-type investors, that the memory parameter associated with the forecasting of price return variance does not affect the local dynamics around equilibria.
} 
where

$$
\Phi_{n}\left(x_{1}, x_{2}, \ldots, x_{N} ; \varphi_{1}, \varphi_{2}, \ldots, \varphi_{N-1} ; e ; r\right)=\varphi_{n} \frac{1+(r+e) x_{n}}{1+(r+e) \sum_{m=1}^{N} \varphi_{m} x_{m}}
$$

for $n \in\{1, \ldots, N-1\}$ and where the price return $r_{t+1}$ is

$$
r_{t+1}=\frac{\sum_{n=1}^{N} \varphi_{t, n}\left(f_{n}\left(y_{t, n}, z_{t, n}\right)\left(1+e_{t+1} x_{t, n}\right)-x_{t, n}\right)}{\sum_{n=1}^{N} \varphi_{t, n} x_{t, n}\left(1-f_{n}\left(y_{t, n}, z_{t, n}\right)\right)}
$$

with

$$
\varphi_{t, N}=1-\sum_{n=1}^{N-1} \varphi_{t, n}
$$

The equations are arranged in four separate blocks: $\mathcal{X}, \mathcal{Y}, \mathcal{Z}$ and $\mathcal{W}$. The $N$ equations in block $\mathcal{X}$ give the investment choices of the $N$ agents, according to (2.8). Blocks $\mathcal{Y}$ and $\mathcal{Z}$ contain the $N$ recursive relations (2.10) describing the evolution of the EWMA estimates of return and its variance, respectively, for the different agents. The evolution of the wealth shares is described by the equations in block $\mathcal{W}$; notice that the number of independent wealth shares in the system is $N-1$. The evolution of price return is provided by (4.3) in accordance with (2.13).

The rest of this Section is devoted to the analysis of the deterministic skeleton of (4.1) obtained replacing the yield process $\left\{e_{t}\right\}$ by its mean value $\bar{e}$.

\subsection{Determination of the equilibria}

The characterization of the fixed points of the system (4.1) is, in many respects, similar to the single agent case discussed in Sec. 3. Let

$$
\boldsymbol{x}^{*}=\left(x_{1}^{*}, \ldots, x_{N}^{*} ; y_{1}^{*}, \ldots, y_{N}^{*} ; z_{1}^{*}, \ldots, z_{N}^{*} ; \varphi_{1}^{*}, \ldots, \varphi_{N-1}^{*}\right)
$$

denote an equilibrium and let $r^{*}$ be the associated equilibrium return. We introduce the following

Definition 4.1. Agent $n$ is said to "survive" in $\boldsymbol{x}^{*}$ if his wealth share is strictly positive, $\varphi_{n}^{*}>0$. Agent $n$ is said to "dominate" the economy, if he is the only survivor in the economy, so that $\varphi_{n}^{*}=1$.

One can recognize the parallel between our definition above and the framework developed in DeLong et al. (1991). Indeed, we adopt here the deterministic version of the concepts of survival and dominance used in that paper.

The following statement characterizes all possible equilibria of system (4.1).

Proposition 4.1. Let $\boldsymbol{x}^{*}$ be a fixed point of the deterministic skeleton of system (4.1). Then

$$
y_{n}^{*}=r^{*}, \quad z_{n}^{*}=0, \quad x_{n}^{*}=f_{n}\left(r^{*}, 0\right) \quad \forall n \in\{1, \ldots, N\},
$$

and the following three mutually exclusive cases are possible:

(i) Survival of a single agent. In $\boldsymbol{x}^{*}$ only one agent survives and, therefore, dominates the economy. Without loss of generality we can assume this agent to be agent 1 so that for the equilibrium wealth shares one has

$$
\varphi_{n}^{*}= \begin{cases}1 & \text { if } n=1 \\ 0 & \text { if } n>1\end{cases}
$$


The equilibrium return $r^{*}$ satisfies the following equation

$$
l\left(r^{*}\right)=f_{1}\left(r^{*}, 0\right)
$$

and coincides with the growth rate of the wealth of the survivor, $\rho_{1}^{*}=r^{*}$.

(ii) Survival of many agents. In $\boldsymbol{x}^{*}$ more than one agent survives. Without loss of generality we can assume that the survivors are the first $k$ agents (with $k>1$ ) so that the equilibrium wealth shares satisfy

$$
\left\{\begin{array}{ll}
\varphi_{n}^{*} \in(0,1) & \text { if } n \leq k, \\
\varphi_{n}^{*}=0 & \text { if } n>k
\end{array} \quad, \quad \sum_{n=1}^{k} \varphi_{n}^{*}=1 .\right.
$$

The equilibrium return $r^{*}$ satisfies the following $k$ equations

$$
l\left(r^{*}\right)=f_{n}\left(r^{*}, 0\right) \quad \forall n \in\{1, \ldots, k\} \quad,
$$

so that the first $k$ agents possess, at equilibrium, the same investment share $x_{1 \diamond k}^{*}=l\left(r^{*}\right)$. The wealth growth rates of the survivors are equal to the price return, i.e. $\rho_{n}^{*}=r^{*}$ for $n \leq k$.

(iii) "No arbitrage" with many survivors. In $x^{*}$ the investment shares and wealth shares of the agents satisfy

$$
\sum_{n=1}^{N} x_{n}^{*} \varphi_{n}^{*}=0 \quad \text { and } \quad \sum_{n=1}^{N} \varphi_{n}^{*}=1
$$

while equilibrium return $r^{*}=-\bar{e}$. The wealth growth rate is zero for all the agents.

Proof. See appendix F.

Strictly speaking, item $(i)$ of the previous Proposition can be seen as a particular case of item (ii). Nevertheless, the nature of the two situations is different. In the first case, when a single agent survives, Proposition 4.1 defines a precise value for each component of the equilibrium $\boldsymbol{x}^{*}$, so that a single point is uniquely determined. In the second case, when many agents survive, there is a residual degree of freedom in the definition of the equilibrium: while investment shares $x^{*}$ and estimators values $y^{*}$ and $z^{*}$ are uniquely defined, the only requirement on the equilibrium wealth shares of the survivors is the fulfillment of the second equality in (4.8). Consequently, one immediately has the following

Corollary 4.1. Consider the deterministic skeleton of system (4.1). If it possesses one equilibrium $\boldsymbol{x}^{*}$ with $k$ survivors, it possesses a $k-1$-simplex of equilibria with $k$-survivors constituted by all the points obtained from $\boldsymbol{x}^{*}$ through a change in the relative wealths of the survivors.

Thus, Proposition 4.1(ii) does not define a single equilibrium point, but an infinite set of equilibria. If the survivors are the first $k$ agents as in (4.8), this set can be written as

$$
\left\{(x_{1}^{*}, \ldots, x_{N}^{*} ; \underbrace{r^{*}, \ldots, r^{*}}_{N} ; \underbrace{0, \ldots, 0}_{N} ; \varphi_{1}, \ldots, \varphi_{k}, \underbrace{0, \ldots, 0}_{N-1-k}) \mid \sum_{j=1}^{k} \varphi_{j}=1, \varphi_{j} \geq 0\right\} .
$$

The particular fixed point eventually chosen by the system will depend on the initial conditions. We will see below that the partially indeterminate nature of the many survivors equilibria will have a major effect also on their stability. 
The differences among the first two items of Proposition 4.1 do not concern exclusively the geometrical nature of the locus of equilibria. Indeed, while in the first case no requirements are imposed on the behavior of the investment function of the different agents, in the second type of solutions all the investment shares $x_{1}^{*}, \ldots, x_{k}^{*}$ must at the same time be equal to a single value $x_{1 \diamond k}^{*}$. Thus, the equilibrium with $k>1$ survivors exists only in the particular case in which $k$ investment functions $f_{1}, \ldots, f_{k}$ satisfy this restriction. This implies that an economy composed of $N$ agents having generic, so to speak "randomly defined", investment functions, has probability zero of displaying any equilibrium with multiple survivors. In other words, the many survivors equilibria are non-generic.

While the first two types of equilibria derived in Proposition 4.1 are related to single agent equilibria, the equilibria described in item (iii) are completely new. In these equilibria many agents survive, and their investment and wealth shares are "balanced" in such a way that the capital gain and the dividend yield offset each other so that the riskless and the risky assets have the same expected return. As opposed to the situation described in Proposition 4.1(ii), these are generic equilibria with many survivors. It is easy to check that the only requirement for the existence of no-arbitrage equilibrium is the co-existence of two agents in the market, one with positive and one with negative investment share ${ }^{9}$. Furthermore, if this condition is satisfied, then all no-arbitrage equilibria belong to the following $N-2$-dimensional manifold

$$
\left\{(x_{1}^{*}, \ldots, x_{N}^{*} ; \underbrace{-\bar{e}, \ldots,-\bar{e}}_{N} ; \underbrace{0, \ldots, 0}_{N} ; \varphi_{1}, \ldots, \varphi_{N-1-k}) \mid \sum_{j=1}^{N} \varphi_{j}=1, \sum_{j=1}^{N} \varphi_{j} x_{j}^{*}=0, \varphi_{j} \geq 0\right\} .
$$

The geometrical interpretation of the market equilibria presented in Section 3 can be extended to illustrate how equilibria with many agents are determined. As an example consider again the left panel in Fig. 1 and suppose that the two investment functions shown there belong to two agents who are simultaneously trading in the market. According to Proposition 4.1 all possible equilibria are the intersections of the different investment functions with the EML (cf. (4.7) and (4.9)), which, however, has to be supplemented by the vertical line at $-\bar{e}$ to illustrate also the no-arbitrage equilibria. In this example there are four equilibria with single survivor. In two of them $\left(S_{1}\right.$ and $\left.U_{1}\right)$ the first agent, with non-linear investment function, survives such that $\varphi_{1}^{*}=1$ (and obviously $\varphi_{2}^{*}=0$ ). In the other two equilibria $\left(S_{2}\right.$ and $\left.U_{2}\right)$ the second agent, with linear investment function, survives so that in these points $\varphi_{1}^{*}=0$. In each equilibrium, the intersection of the investment function of the surviving agent with the EML gives both the equilibrium return and the equilibrium investment share of the survivor. The equilibrium investment share of the non-surviving agent can be found, in accordance with the last relation in (4.5), as the intersection of his/her own investment function with the vertical line passing through the equilibrium return. Since the two investment functions shown in Fig. 1 do not possess common intersections with the EML, the equilibria of the second type are impossible in this case. There exist, however, one no-arbitrage equilibrium with $r^{*}=-\bar{e}$ which geometrically can be represented by the two points $A_{1}$ and $A_{2}$ showing the corresponding investment shares of the agents. The equilibrium wealth shares of the two agents can be derived from (4.10).

An example of investment functions which allow for multiple survivors equilibria is given in the left panel of Fig. 2. The abscissa of the common intersections of the different investment functions with the EML defines the return in the case of equilibria with multiple survivors. In each equilibrium, all the survivors invest the same share of wealth $x_{1 \diamond k}^{*}$ in the risky asset, determined as the ordinate of the intersection. The wealth shares of the survivors should satisfy (4.8). The non surviving agents (like the agent with investment function I in equilibrium $S_{2}$ or the agent with investment function III in equilibrium $U_{1}$ ) have zero wealth shares. Their investment shares are the intersections of their investment functions with the vertical line passing through the equilibrium return.

\footnotetext{
${ }^{9}$ Essentially, this is the reason why no-arbitrage equilibria do not exist in the single agent case.
} 

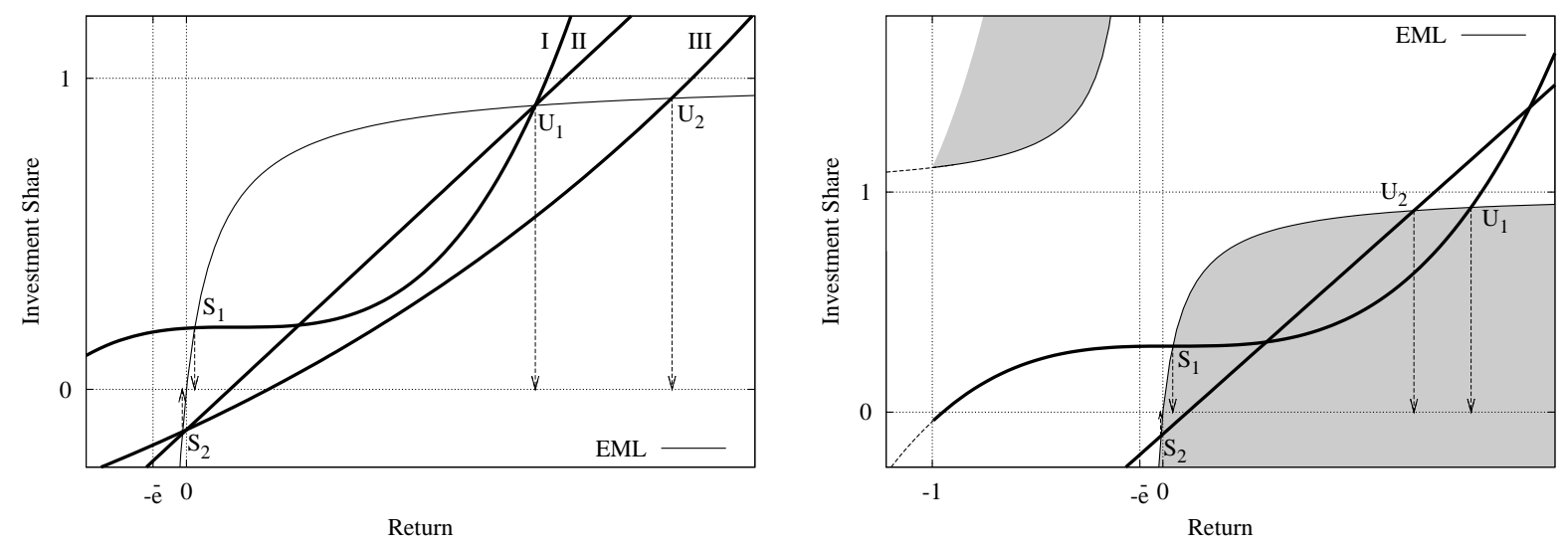

Figure 2: Equilibria and stability for the many agents system. Left panel: Non-generic situation with 3 agents operating in the market. In equilibria $S_{2}$ and $U_{1}$ two agents survive. Right panel: Generic situation with 2 agents operating in the market. The region where condition (4.12) is satisfied is shown in gray.

\subsection{The stability conditions of the equilibria}

Among the different equilibria characterized in the previous Section, which are the ones eventually selected by the market? This Section answers this question presenting the results of the local stability analysis for all possible equilibria. The first Proposition below provides the stability region in the parameter space for the generic case of one single survivor. The non-generic case of many survivors is addressed in the second Proposition, where the destabilizing effect of the existence of an entire hyperplane of equilibria is revealed. Finally, in the third Proposition we derive the stability conditions for no-arbitrage equilibria with many survivors. The derivation of these Propositions requires quite cumbersome algebraic manipulations and we refer the reader to Appendix $G$ for the intermediate Lemmas and the final proofs. The discussion concerning economic interpretation of the results, analysis of their consequences for the aggregate behavior of the system and comparison with other contributions to the literature are postponed to the next Section.

For the generic case of a single survivor equilibrium we have the following

Proposition 4.2. Let $\boldsymbol{x}^{*}$ be a fixed point of the deterministic skeleton of system (4.1) associated with a single survivor equilibrium. Without loss of generality we can assume (4.6), i.e. that the first agent is the survivor. Then $\boldsymbol{x}^{*}$ is (locally) asymptotically stable if

$$
\frac{f_{1, y}^{\prime}\left(r^{*}, 0\right)}{l^{\prime}\left(r^{*}\right)} \frac{1}{r^{*}}<\frac{1}{1-\lambda_{1}} \quad, \quad \frac{f_{1, y}^{\prime}\left(r^{*}, 0\right)}{l^{\prime}\left(r^{*}\right)}<1 \quad, \quad \frac{f_{1, y}^{\prime}\left(r^{*}, 0\right)}{l^{\prime}\left(r^{*}\right)} \frac{2+r^{*}}{r^{*}}>-\frac{1+\lambda_{1}}{1-\lambda_{1}}
$$

where $f_{1, y}^{\prime}$ denotes the partial derivative of investment function $f_{1}$ with respect to the expected return, and if

$$
-2-r^{*}<x_{n}^{*}\left(r^{*}+\bar{e}\right)<r^{*}, \quad \forall n>1 .
$$

The equilibrium $\boldsymbol{x}^{*}$ is unstable if at least one of the inequalities in (4.11) or in (4.12) holds with the (strict) opposite sign.

The system exhibits a Neimark-Sacker bifurcation if the first inequality in (4.11) becomes an equality, a fold bifurcation if the second inequality in (4.11) or one of the $N-1$ right-hand inequalities in (4.12) becomes an equality and a flip bifurcation if the third inequality in (4.11) or one of the $N-1$ left-hand inequalities in (4.12) becomes an equality. 
The stability conditions for a generic single-survivor equilibrium contain two requirements. On the one hand, the stable equilibrium should be "self-consistent", i.e. it should remain stable even if any non-surviving agent is removed from the economy. Indeed the three inequalities in (4.11) coincide with the corresponding conditions in (3.7) as if the survivor would operate alone in the market. This is however not enough. A further requirement comes from the inequalities in (4.12). In particular, notice that in those equilibria where $r^{*}>-\bar{e}$, i.e. where the overall wealth of the economy grows ${ }^{10}$, the surviving agent must be the most aggressive one, i.e. the one who, among all agents, invests the highest wealth share in the risky asset. On the other hand, in those equilibria where $r^{*}<-\bar{e}$, i.e. in which the overall wealth of the economy shrinks, the survivor has to be the least aggressive investor.

For the single survivor equilibrium the role of the "memory" parameter $\lambda$ is similar to what found for the single agent case in Proposition 3.4. The equilibrium stability domain increases with the value of the survivor's $\lambda$. In the case of heterogeneous agents, however, the scope of this statement is restricted by an important caveat: if the additional condition in (4.12) is not satisfied, the equilibrium remains unstable irrespectively of the value of $\lambda$.

Let us revert once again to the EML "plot" to obtain a geometrical illustration of the many-agents stability conditions. In the right panel of Fig. 2 we draw two investment functions, one linear and one non-linear. The region where condition (4.12) is satisfied is reported in gray. Suppose that the market is populated by two agents whose investment decisions are respectively described by these two functions. Notice that they are the same functions appearing in the left panel of Fig. 1 and that were discussed in Section 3.1. Proposition 4.1 allowed us to identify four possible equilibria with one survivor: $S_{1}, S_{2}, U_{1}$ and $U_{2}$. First, notice that the dynamics of the two-agent system cannot be attracted by $U_{1}$ or $U_{2}$. Since these equilibria were unstable in the respective single-agent cases, they cannot be stable when both agents are present in the market. Assume that $S_{1}$ and $S_{2}$ would be stable equilibria if the first and the second function, respectively, were present alone in the market. Then, from Proposition 4.2, it follows that $S_{1}$ is the only stable equilibrium of the system with two agents. Notice, indeed, that for a value of $r$ equal to the abscissa of $S_{1}$, i.e. equal to the equilibrium return, the linear investment function of the non-surviving agent passes below the investment function of the surviving agent and belongs to the gray area. On the contrary, in the abscissa of $S_{2}$, the investment function of the non-surviving agent is higher and does not belong to the gray area. Consequently, the latter equilibrium is unstable.

Let us move now to consider the non-generic case, when $k$ different agents survive in the equilibrium. The following applies

Proposition 4.3. Let $\boldsymbol{x}^{*}$ be a fixed point of the deterministic skeleton of system (4.1) belonging to a $k-1$-dimensional manifold of the $k$-survivors equilibria defined by (4.5), (4.8) and (4.9).

The fixed point $\boldsymbol{x}^{*}$ is never hyperbolic and, consequently, never (locally) asymptotically stable. Its non-hyperbolic submanifold is the $k-1$-simplex defined in Corollary 4.1.

The equilibrium $\boldsymbol{x}^{*}$ is (locally) stable if

(i) all the roots of the following polynomial are inside the unit circle

$$
\mu \prod_{j=1}^{k}\left(\lambda_{j}-\mu\right)+\frac{\left(1+r^{*}\right) \mu-1}{x_{1 \diamond k}^{*}\left(1-x_{1 \diamond k}^{*}\right)} \sum_{j=1}^{k}\left(\varphi_{j}^{*} f_{j, y}^{\prime}\left(1-\lambda_{j}\right) \prod_{i=1, i \neq j}^{k}\left(\lambda_{i}-\mu\right)\right),
$$

where $f_{j, y}^{\prime}$ denotes the partial derivative of investment function $f_{j}$ with respect to the estimated return y;

\footnotetext{
${ }^{10}$ The equilibrium growth rate of the wealth of the economy coincides, of course, with the wealth growth rate of survivor and equal, therefore, to $r^{*}$. If $r^{*}>0$, then the wealth of the economy is positive and growing. If $r^{*} \in(-\bar{e}, 0)$, the economy has negative growth rate but also negative wealth. Thus, also for these equilibria the economy is growing.
} 
(ii) the equilibrium investment shares of the non-surviving agents satisfy

$$
-2-r^{*}<x_{n}^{*}\left(r^{*}+\bar{e}\right)<r^{*}, \quad k<n \leq N .
$$

The equilibrium $\boldsymbol{x}^{*}$ is unstable if at least one of the roots of the polynomial in (4.13) is outside the unit circle or if at least one of the inequalities in (4.14) holds with the (strict) opposite sign.

The non hyperbolic nature of the many-survivors equilibria is a direct consequence of their nonunique specification in Proposition 4.1(ii). However, the motion of the system along the $k-1$ dimensional subspace consisting of the continuum of equilibria leaves the aggregate properties of the system unaltered.

The stability conditions for the many survivors equilibria generalize the stability conditions derived in Proposition 4.2 for the single survivor case. Indeed, the constraints on the investment shares of the non-surviving agents (4.14) are identical to (4.12) and the condition in the first item of Proposition reduces to the inequalities (4.11) when $k=1$. This condition is simplified when survivors possess homogeneous EWMA estimators.

Corollary 4.2. Let $\boldsymbol{x}^{*}$ be a fixed point of the deterministic skeleton of system (4.1) belonging to a $k-1$-dimensional manifold of $k$-survivors equilibria. If all the surviving agents possess identical values of the EWMA parameter

$$
\lambda=\lambda_{1}=\cdots=\lambda_{k}
$$

the condition ( $i$ ) in Proposition 4.3 becomes

$$
\frac{\left\langle f_{y}^{\prime}\right\rangle}{l^{\prime}\left(r^{*}\right)} \frac{1}{r^{*}}<\frac{1}{1-\lambda} \quad, \quad \frac{\left\langle f_{y}^{\prime}\right\rangle}{l^{\prime}\left(r^{*}\right)}<1 \quad, \quad \frac{\left\langle f_{y}^{\prime}\right\rangle}{l^{\prime}\left(r^{*}\right)} \frac{2+r^{*}}{r^{*}}>-\frac{1+\lambda}{1-\lambda},
$$

where $\left\langle f_{y}^{\prime}\right\rangle=\sum_{j=1}^{k} \varphi_{j}^{*} f_{j, y}^{\prime}$.

The inequalities in (4.15) are identical to the stability conditions for the single-agent case, except that one has to weight the derivatives computed in the fixed point with the wealth shares of the surviving agents. Interestingly, this implies that an agent with an investment function generating unstable equilibria if present alone in the market, may survive with the same function in a stable non-generic equilibrium if other agents, with stable functions, possess, at equilibrium, high enough wealth shares.

Finally, let us analyze the local stability of no-arbitrage equilibria with $r^{*}=-\bar{e}$. We consider a generic situation and allow some agents to have zero wealth share. Without loss of generality we can assume that the first $k$ agents survive, where $k \leq N$.

Proposition 4.4. Let $\boldsymbol{x}^{*}$ be a fixed point of the deterministic skeleton of system (4.1) belonging to an $N-2$-dimensional manifold of $k$-survivors equilibria defined by (4.5) and (4.10).

If $N \geq 3$, the fixed point $\boldsymbol{x}^{*}$ is non hyperbolic and, consequently, non (locally) asymptotically stable. The equilibrium $\boldsymbol{x}^{*}$ is (locally) stable if all the roots of the following polynomial are inside the unit circle

$$
\mu \prod_{j=1}^{k}\left(\lambda_{j}-\mu\right)+\frac{1-\mu}{\left\langle x^{2}\right\rangle} \sum_{j=1}^{k}\left(\varphi_{j}^{*} f_{j, y}^{\prime}\left(1-\lambda_{j}\right) \prod_{i=1, i \neq j}^{k}\left(\lambda_{i}-\mu\right)\right),
$$

where $f_{j, y}^{\prime}$ denotes the value of the partial derivative of investment function $f_{j}$ with respect to the expected return $y$ in the point $(-\bar{e}, 0)$, and $\left\langle x^{2}\right\rangle=\sum_{n=1}^{k} \varphi_{n}^{*} x_{n}^{* 2}$.

The equilibrium $\boldsymbol{x}^{*}$ is unstable if at least one of the roots of the polynomial in (4.16) is outside the unit circle. 
The stability of equilibria now depends exclusively on the polynomial (4.16). For specific investment functions this polynomial can be simplified and explicit stability conditions derived. For instance, when all investment functions are flat at $-\bar{e}$, it is easy to see that the no-arbitrage equilibrium (if it exists) is always stable. Another simplification is obtained when survivors have homogeneous EWMA estimator.

Corollary 4.3. Let $\boldsymbol{x}^{*}$ be a no-arbitrage fixed point of the deterministic skeleton of system (4.1) belonging to an $N-2$-dimensional manifold of $k$-survivors equilibria. If all the surviving agents possess identical values of the EWMA parameter

$$
\lambda=\lambda_{1}=\cdots=\lambda_{k}
$$

the condition in Proposition 4.4 becomes

$$
\frac{\left\langle f_{y}^{\prime}\right\rangle}{\left\langle x^{2}\right\rangle}>-\frac{1}{1-\lambda} \quad, \quad \frac{\left\langle f_{y}^{\prime}\right\rangle}{\left\langle x^{2}\right\rangle}<\frac{1}{2} \frac{1+\lambda}{1-\lambda}
$$

where $\left\langle f_{y}^{\prime}\right\rangle=\sum_{j=1}^{k} \varphi_{j}^{*} f_{j, y}^{\prime}(-\bar{e}, 0)$.

According to this Corollary, for sufficiently large $\lambda$ the no-arbitrage equilibrium becomes always stable .

\section{Examples and Applications}

In this Section we present two examples of how the results obtained above, and in particular the use of the EML "plot", can be applied to the analysis of several aspects of some heterogeneous agent models recently discussed in the literature. In Section 5.1 we consider investment functions based on the mean-variance approximate solution of the expected utility maximization problem with power utility function. We discuss the effects of different agent-specific parameters on the local and global behavior of the system, stressing the similarity with previous investigations. In Section 5.2 we take a broader approach and present a general discussion of the principles according to which, in a heterogeneous environment, the dynamics of the market selects the investment functions that eventually dominate the economy.

\subsection{Investment functions from an approximation to power utility}

As mentioned before, among the different agents' specifications which are allowed inside our framework, there are the trading behaviors derived from the maximization of expected CRRA utility. Consider the problem that a typical agent faces at period $t$ before trade takes place

$$
\max _{x_{t}} \mathrm{E}\left[U\left(W_{t+1}\right)\right], \quad \text { s.t. } \quad W_{t+1}=W_{t}\left(1+x_{t}\left(R_{t+1}+D_{t+1} / P_{t}\right)\right)
$$

where $U(W)=\left(W^{1-\gamma}-1\right) /(1-\gamma)$ is a power utility function with the relative risk aversion $\gamma>0$, and $R_{t+1}$ is the return in terms of unscaled price as in (2.7). It is well known that the solution $x_{t}$ of (5.1) is independent of the agent's current wealth $W_{t}$ while it depends on agent's expectations about next period (gross) return. Let us assume that these expectations are expressed in terms of EWMA estimators of price return and its variance under some ancillary assumption about the functional form of the conditional probability density of price returns. Then the solution of the problem belongs to 
the set of investment functions satisfying Assumption 2. Unfortunately, the explicit expression of the corresponding investment function cannot be derived for reasonable conditional distributions of returns (e.g. normally distributed returns). In order to obtain a tractable analytic expression one has to turn to some approximation. Following Chiarella and He (2001) we consider here the meanvariance approximation ${ }^{11}$ of the solution of (5.1) that in terms of rescaled variables (2.4) becomes

$$
x_{t}=\frac{1}{\gamma\left(1+r_{f}\right)} \frac{E_{t-1}\left[r_{t+1}+e_{t+1}\right]}{V_{t-1}\left[r_{t+1}+e_{t+1}\right]},
$$

where $E_{t-1}$ and $V_{t-1}$ stand for the agent's expectations about mean and variance of the (rescaled) gross return, respectively. Consistent with Assumptions 1 and 2, the first two moments $\bar{e}$ and $\sigma_{e}^{2}$ of the i.i.d. yield process are known to the traders, while the expectations about return depend on the past market history through the EWMA estimators. More precisely, we assume the following expressions

$$
\begin{aligned}
& E_{t-1}\left[r_{t+1}+e_{t+1}\right]=\delta+d\left(y_{t-1}+\bar{e}\right), \\
& V_{t-1}\left[r_{t+1}+e_{t+1}\right]=z_{t-1}+\sigma_{e}^{2} .
\end{aligned}
$$

The linear functional form in (5.3) is equivalent to the specification analyzed in Chiarella and He (2001) and allows for a simple interpretation: the parameter $\delta>0$ can be interpreted as a risk premium, while the extrapolation parameter $d$ characterizes the relation of present investment choice to past market dynamics. Using this parameter one can distinguish between different stylized types of trading behavior. A trader with $d=0$ can be identified with a fundamentalist, since his investment choice is unaffected by the past return realizations. For $d \neq 0$ the agent is a chartist. Specifically, he is a trend follower if $d>0$ and a contrarian if $d<0$. Higher values of the past returns lead to higher investment choices for trend followers and to lower investment choices for contrarians. Plugging (5.3) and (5.4) into (5.2) one gets the investment function

$$
f(y, z)=\frac{1}{\gamma\left(1+r_{f}\right)} \frac{\delta+d(y+\bar{e})}{z+\sigma_{e}^{2}} .
$$

As the results of Section 3 imply, the location of the equilibria and their stability analysis can be performed considering the restriction of (5.5) to the line $z=0$. The restricted investment function possesses a simple linear form

$$
f(y, 0)=\tilde{\delta}+\tilde{d}(y+\bar{e}), \quad \text { with } \quad \tilde{\delta}=\frac{\delta}{\gamma\left(1+r_{f}\right) \sigma_{e}^{2}}, \quad \tilde{d}=\frac{d}{\gamma\left(1+r_{f}\right) \sigma_{e}^{2}}
$$

Notice that the use of a more complicated function of $z$ for the variance expectation in (5.4) as for example Equation 3.2 in Chiarella and $\mathrm{He}$ (2001), would only lead to a rescaling of the parameters in (5.6), proportional to the value of this function computed at $z=0$.

Below we provide some examples on the use of the EML "plot" to investigate the role of the parameters of (5.6) in the definition of the number, location and stability of the equilibria. We do not present a complete analysis, but starting from these examples it is easy to develop further the formal machinery and, in particular, re-obtain the results in Chiarella and $\mathrm{He}$ (2001) for the case of one and two-agent economies ${ }^{12}$.

\footnotetext{
${ }^{11}$ This is not the only mean-variance approximation, see e.g. Campbell and Viceira (2002).

${ }^{12}$ The only difference between our investment functions and the ones in Chiarella and He (2001) is that they use the equally weighted estimators computed on the basis of the past $L>0$ returns, instead of our EWMA estimators. Such a difference is not important for the determination of the equilibria, however, since in any fixed point the value of the two estimators coincides and is equal to the equilibrium return. The stability analysis is performed in Chiarella and He (2001) only for the case $L=1$, which is a particular case of our framework corresponding to $\lambda=0$.
} 

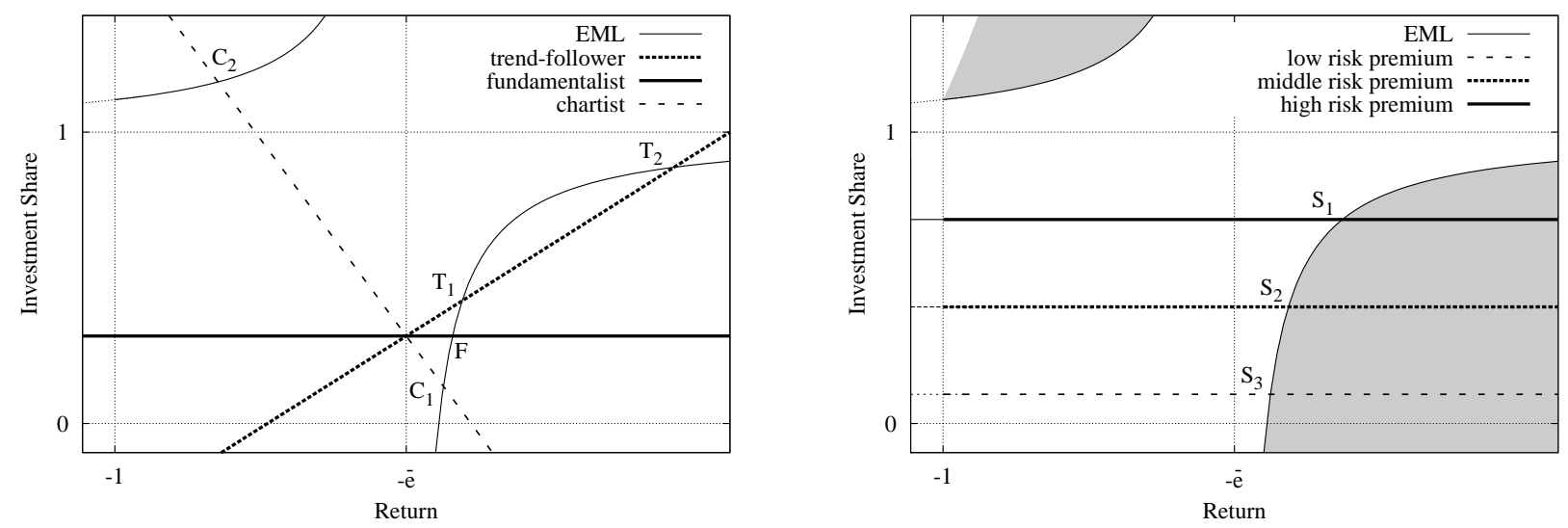

Figure 3: Equilibria in the market with investment functions based on the mean-variance solution of the utility-maximization problem. Left panel: Three typical agent's behaviors. Right panel: Equilibria with three different fundamentalists. $S_{1}$ is the only stable equilibrium.

In the left panel of Fig. 3 three investment functions (5.6) are shown with the same value of $\tilde{\delta} \in(0,1)$ and different values of $\tilde{d}$. The horizontal investment function corresponds to $\tilde{d}=0$, i.e. to the fundamentalist behavior. The fundamentalist function has one equilibrium, denoted by $F$. From the picture it is obvious that varying the value of $\tilde{d}$, the amount of wealth invested in the risky asset, the horizontal investment function always has (apart the forbidden value $x=1$ ) a unique equilibrium (cf. Chiarella and He (2001), first item of Proposition 3.1).

Now consider values of $\tilde{d}$ below zero, associated with contrarian behavior. The equilibrium in the right branch of the EML, denoted by $C_{1}$, moves toward the lower values of $x^{*}$ and $r^{*}$. At the same time, a second equilibrium, denoted by $C_{2}$, appears as the intersection of the investment function with the left branch of the EML. This second equilibrium is characterized by a low value of the price return $r^{*}<-\bar{e}$. Notice that it belongs to the feasible part of the left branch (i.e. leads to positive equilibrium prices) only if $\tilde{d}$ is small enough. It follows that the contrarian investment function always possesses two equilibria, but one of them may be unfeasible (cf. Chiarella and He (2001), second item of Proposition 3.1).

Finally, for $\tilde{d}>0$, the agent is a trend-follower. For low enough values of $\tilde{d}$, his/her investment function possesses two intersections, denoted by $T_{1}$ and $T_{2}$, with the right branch of the EML. With the increase of the value of $\tilde{d}$, the equilibrium return associated with the first intersection increases while the one associated with the second intersection decreases until they coincide and then disappear, in the point where the investment function is tangent to the EML. If one considers relatively high values of the parameter $\tilde{\delta}$ and of the slope $\tilde{d}$, the trend follower function can also possess one or two intersections with the left branch of the EML. Thus, the trend-followers investment function possesses 0, 1 or 2 equilibria (cf. Chiarella and He (2001), third item of Proposition 3.1).

Concerning equilibria stability, from Proposition 3.4 we immediately see that the equilibrium $F$ of the fundamentalist is always stable, while the equilibrium $T_{2}$ of the trend-followers is always unstable, because it violates the second inequality in (3.7). The other types of equilibria (like $T_{1}, C_{1}$ and $C_{2}$ in the left panel of Fig. 3) become stable with a high enough value of $\lambda$.

Let us now consider a model with heterogeneous agents. We confine our illustration to the case of several fundamentalists. The extension to other scenarios is straightforward. In the right panel of Fig. 3 we draw three fundamentalist investment functions with different values of the risk premia $\tilde{\delta}$. There are three "generic" equilibria: $S_{1}, S_{2}$ and $S_{3}$. It follows from Proposition 4.2 
that only one of them is stable, namely $S_{1}$. This is the only equilibrium in which the survivor is the most aggressive trader. Thus, among different fundamentalists, it is the one with the highest risk premium who survives (cf. Chiarella and He (2001), Corollary 4.3). Since $\tilde{\delta}$ is inversely proportional to the risk aversion parameter $\gamma$ one can say that among the fundamentalists with different risk aversion coefficients the one with the smallest coefficient, ceteris paribus, survives and dominates the economy. This is not a peculiar feature of the fundamentalist investment function, however. In general terms, one can describe the effect of an increase (decrease) in the agent's risk aversion as a downward (upward) shift of his/her investment function. The shift implies that a different quantity (lower or higher) of the risky asset is held in the agent's portfolio for the same level of expected price return. Because of (4.12) or (4.14) the shift of one investment function can have a strong destabilizing effect on the equilibria of the other functions, and possibly disrupt already established dominant positions. The ensuing out-of-equilibrium dynamics can eventually drive the market towards a new equilibrium in which the agent who increased his/her risk aversion dominates the economy. This general property seems to be in remarkable agreement with the following result suggested by a simulation model in Zschischang and Lux (2001) (p.568, 569):

Looking more systematically at the interplay of risk aversion and memory span, it seems to us that the former is the more relevant factor, as with different [risk aversion coefficients] we frequently found a reversal in the dominance pattern: groups which were fading away before became dominant when we reduced their degree of risk aversion...

...It also appears that when adding different degrees of risk aversion, the differences of time horizons are not decisive any more, provided the time horizon is not too short.

In the model by Zschischang and Lux the CRRA-agents have limited memory spans (time horizons) and forecast the next return as the average of past realized returns. Since different lengths of the memory spans can be approximated by different values of $\lambda$, the result described in the above quotation is exactly what we would expect according to our Proposition 4.2. Sufficiently long time horizons are needed in order to satisfy condition (4.11). Then, the survivor is determined solely by the conditions in (4.12) which hold for the agent with the highest risk aversion.

The above discussion seems to suggest that the market tends to prefer "higher" investment functions. In the proposed multi-agent example the economy always ends up in the equilibrium with the highest possible return. That is, the dynamics endogenously select the best aggregate outcome. As we will discuss below, this is not always the case.

\subsection{On the competition among strategies}

The stability analysis performed in Section 4.3 provides important information about the asymptotic conditions and the relative performances of the different traders interacting in the market. Inspired by the famous Friedman hypothesis one could expect that if there is an agent who makes better use of the information revealed by the trading activity and of the common and perfect knowledge about the fundamental process, this more "rational" trader will outperform the others and ultimately drive them out of the market. How and to what degree does this hypothetical result prove to be valid within our framework? A different but related question is whether and to what extent the "dynamic" selection mechanism, which leads one type of agents to survive and possibly dominate the market, is beneficial to the economy as a whole, thus generating a higher growth rate of the total wealth.

Let us consider a stable many agents equilibrium with price return $r^{*}$. According to the results of the stability analysis, the wealth return of all survivors is equal to $r^{*}$, so that $r^{*}$ is also the asymptotic growth rate of the total wealth. At the same time, the wealth growth rates of the non surviving agents 

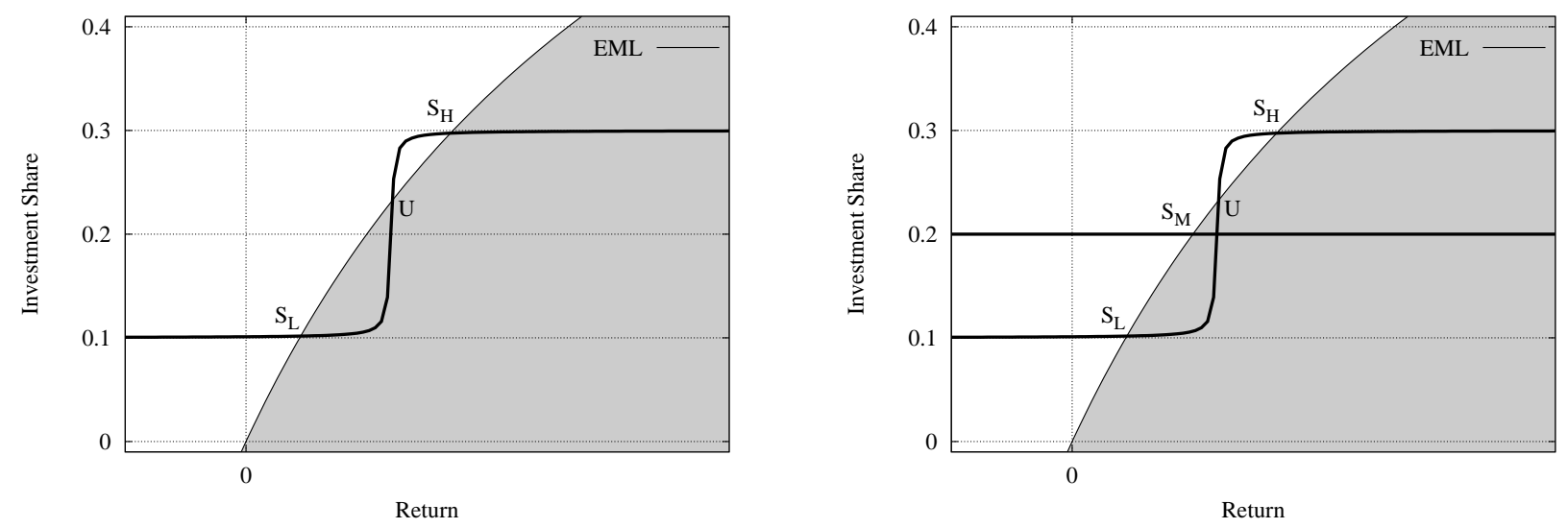

Figure 4: Left panel: A non-linear investment function leading to multiple equilibria. $S_{H}$ and $S_{L}$ are stable while $U$ is unstable. Right panel: The same non-linear investment function together with a constant investment function. $S_{H}$ and $S_{M}$ are stable while $S_{L}$ and $U$ are unstable.

are lower than $r^{*}$. Then, if they were surviving, and consequently were affecting the dynamics of the total wealth, the whole economy would grow at a lower rate. To put the same statement in negative terms, the economy will never end up in an equilibrium where its growth rate is lower than it would be if the survivor(s) were substituted by some other agent(s). One could see in this result an optimal selection principle since it suggests that the market endogenously selects the best aggregate outcome. This result is in line with the intuitive idea that in a model with endogenous wealth dynamics, the agent who invests more in the growing asset increases his/her influence and, eventually, dominates those traders who invest less. Our analysis confirms in part this intuition, but also highlights two important limitations. First, the optimal selection principle does not apply to the whole set of equilibria, but only to the subset formed by the equilibria associated with stable fixed points in the single agent case. For instance, with the investment functions shown in the right panel of Fig. 2, the market will never end up in $U_{1}$, even if this is the equilibrium with the highest possible return, since this equilibrium would not be stable if the survivor were present alone in the market. Second, the possibility of having multiple stable equilibria, even with one single trader, implies that the optimal selection principle has only a local character: the economy does not necessarily converge to the stable equilibrium with the highest possible return. Consider, for instance, the nonlinear investment function shown in the left panel of Fig. 4. This function possesses two stable equilibria: $S_{L}$ and $S_{H}$. The ultimate equilibrium selected by the dynamics when this is the only function in the market will depend on the initial conditions; there are no guarantees that the market will end up in $S_{H}$, the equilibrium associated with the higher growth rate of the aggregate wealth.

The implication of this result for the stochastic dynamical system can be seen from the left panel of Fig. 5 where we show the price trajectories for different values of the initial return $r_{0}$ and the yield standard deviation $\sigma_{e}$. If $\sigma_{e}$ is relatively small, the system does not leave the basin of attraction of one equilibrium. For instance, it converges to $S_{H}$ for $r_{0}=0.001$ and to $S_{U}$ for $r_{0}=0$. Conversely, if the standard deviation is relatively high, the dynamics is jumping between two basins of attraction, so that periods with high return are followed by periods with low return and vice versa.

The existence of multiple equilibria is also related to a second important implication of our analysis: the fact that the dominance of one investment function over another is a "local" property and depends on the initial market conditions. Consider a simple case with two heterogeneous traders. Suppose that their investment functions are the ones depicted in the right panel of Fig. 4 so that 

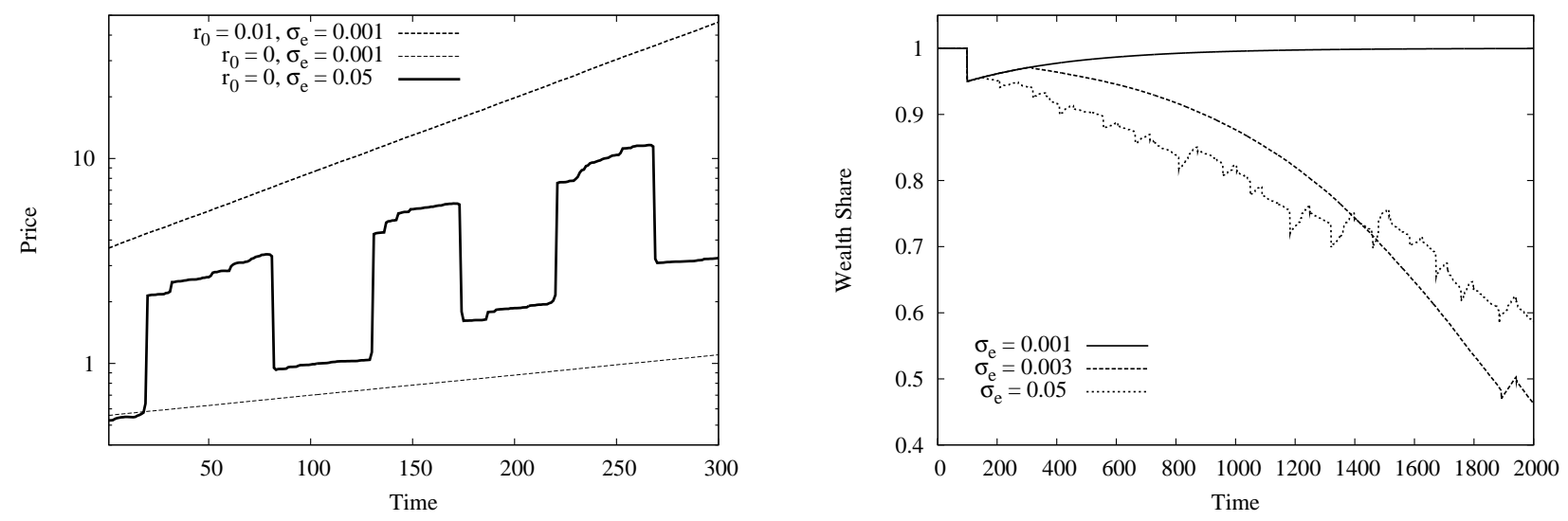

Figure 5: Numerical simulations of the stochastic dynamical system (4.1). The investment functions depicted in Fig. 4 are considered. The yield is log-normally distributed with mean $\bar{e}=0.02$. Left panel: Noise-driven switching between basins of attraction of two different equilibria. Three price trajectories are shown in logscale for the case of single agent with $S$-shaped investment function and for different initial returns $r_{0}$ and standard deviations $\sigma_{e}$. Right panel: Dynamics of the relative wealth share $\varphi_{t, 1}$ of this trader after the entry of the second trader at time $t=100$ with initial wealth share $\varphi_{100,2}=0.05$, for different value of the standard deviations $\sigma_{e}$.

two stable equilibria exist: $S_{M}$ and $S_{H}$. In the former equilibrium the agent with the horizontal investment function dominates, while in the latter equilibrium this agent is dominated by the other agent. Now assume that these two agents enter the market sequentially. It is immediate to see that it is the order in which these two agents enter the market that determines the final aggregate outcome and decides the ultimate survivor.

An example is provided in the right panel of Fig. 5. We initialize the system with a single agent to be at equilibrium $S_{H}$ (see Fig. 4). Then we let the second agent (with linear investment function) enter the market and we show the post-entry dynamics of the wealth share of the incumbent. For small value of $\sigma_{e}$, the entrant is dominated, and, after a relatively short transient, the wealth share of the incumbent returns to 1 . Conversely, if the yield volatility is higher, the second agent, after a relative long transient, asymptotically dominates the market. Notice that when the value of $\sigma_{e}$ is further increased, the system displays the abovementioned phenomenon of switching between neighborhoods of different equilibria. Nevertheless, it spends more time in the basin of attraction of $S_{M}$, so that the first agent is ultimately driven out.

The phenomenon of the displacement of an already dominant strategy by the entry of a new trader is not limited to some peculiar function, rather it is a general property of the model. Indeed, for any given investment function it is always possible to build a second function which makes the equilibria of the first unstable and breaks its possible dominance over the market. For instance, it would be misleading to conclude that the agent who invests the largest share of wealth in the risky security, possesses, due to the endogenous determination of prices, some "permanent" advantage in the speculative struggle. Consider a constant investment function with investment share $x=$ $1-\epsilon, 0<\epsilon \ll 1$. Even if it possesses a stable equilibrium with very high return $r^{*} \sim 1 / \epsilon$, it can be destabilized by a second investment function with constant investment share greater than one. Notice, however, that this second function does not become the winner: indeed, the equilibrium created by this function is unstable in the presence of the first. Rather, the presence of both functions in the market is likely to generate big fluctuations in returns and high volatility.

The impossibility of defining the best or even a "good" investment function independently of the 
set of investment functions present in the market recalls several results in evolutionary finance (see e.g. Hens and Schenk-Hoppé (2005)) and is in tune with the discussion about the limits to arbitrage found in the behavioral finance literature (see e.g. Barberis and Thaler (2003)). It also answers, in negative terms, the question posed at the beginning of this Section about Friedman's hypothesis: any "rational" investment function, for instance derived from a utility maximization procedure, can be destabilized by some "irrational" function, like the one characterized by a constant investment share.

Our results extend to an endogenous price setting the conclusions in DeLong et al. $(1990,1991)$, obtained in simple models with exogenous price dynamics, that rational traders are unable to completely drive the noise investors out of the market. Intuition suggests that in an endogenous price formation setting, when the wealth of the agent feeds back into the model and affects the determination of future price returns, the survival probability of non optimal traders should be increased. We provide a formal basis for this intuitive idea. Indeed, we prove that, inside our endogenous setting, the relative importance of successful (irrational) trading is so high that not only may the rational trader fail to dominate the irrational competitors, but could instead be eventually dominated by them.

\section{Conclusion}

This paper extends previous contributions and presents novel results concerning the characterization and the stability of equilibria in speculative pure exchange economies with heterogeneous adaptive traders. While we mainly focus on theoretical aspects, our results also provide some rigorous background to the growing literature on numerical simulations of artificial agent-based financial markets. Let us briefly review the assumptions we have made and the results we have obtained in order to sketch possible future lines of research.

We considered a simple analytical framework using a minimal number of assumptions ( 2 assets and Walrasian price formation). We modeled agents as speculative traders with individual demand functions proportional to their wealth (CRRA framework). We constrained the agent's portfolio choice to be a function of the past market history, expressed through EWMA estimators of average return and variance. Under a prescribed but arbitrary specification concerning the agents' investment choices we obtained the multi-dimensional dynamical system which describes the feasible dynamics of the economy, i.e. the dynamics for which prices stay always positive. Within this framework, we analyzed the market populated by an arbitrarily large number of heterogeneous agents. Using the Equilibrium Market Line, we found the possible market equilibria, discussed their local stability conditions and the bifurcation types generated by the violation of these conditions. The stability analysis reveals that, even if the existence of multiple stable equilibria implies a local nature for the market selection process and, ultimately, the impossibility of defining any global dominance order relation among agents, in the neighborhood of each equilibrium the market displays a "quasioptimal" behavior leading to the dominance of those investment functions that guarantee a higher growth rate for the whole economy.

However, it should be pointed out that our findings possess different degrees of generality. For instance, the increase in the "memory" of the agent, i.e. the length of the past market history effectively taken into consideration, leads to the stabilization of any single agent equilibrium. This result is possibly related to the particular choice of estimators. Indeed, an analogous property has been found in Bottazzi (2002) within a CARA framework when EWMA estimators were used. The scope and strength of this effect are likely to change when different estimators are used. On the other hand, the fact that the agent's evaluation of endogenous risk does not affect the location and the stability of equilibria is probably a quite robust feature. Indeed, at equilibrium the price return is constant and, 
therefore, any consistent estimator of the variance (or any other central moment) of the conditional return distribution has to converge to zero.

Concerning the existence of three types of equilibria (generic with single survivor, non-generic with many survivors and "no-arbitrage" with many survivors), we can say that preliminary results obtained from the analysis of more general cases (Anufriev and Bottazzi, 2005) confirm the present findings. Nonetheless, in our general framework, numerous specifications of the traders' strategies are possible in addition to the ones already considered. They encompass the inclusion of some form of evaluation of the "fundamental" value of the asset, possibly obtained from a private source of information, of processes of adaptation or co-evolution affecting agents' investment functions, or of strategic behaviors that try to take into consideration the reaction of other market participants to revealed individual choices. Once these more sophisticated behaviors are implemented, the simple picture obtained above is likely to change. In which direction, whether towards a more stable market behavior with a more "optimal" character, or towards a increasingly unstable and volatile dynamics, it is, at this point, hard to say.

\section{APPENDIX: Proofs of Propositions and Lemmas}

\section{A Proof of Proposition 2.1}

Plugging the expression for $w_{t, n}$ from (2.6) into the right-hand side of (2.5), assuming $p_{t-1}>0$ and, consistent with (2.12), $p_{t-1} \neq \sum x_{t, n} x_{t-1, n} w_{t-1, n}$ one gets

$$
\begin{aligned}
p_{t} & =\left(1-\frac{1}{p_{t-1}} \sum_{n=1}^{N} x_{t, n} x_{t-1, n} w_{t-1, n}\right)^{-1}\left(\sum_{n=1}^{N} x_{t, n} w_{t-1, n}+\left(e_{t}-1\right) \sum_{n=1}^{N} x_{t, n} w_{t-1, n} x_{t-1, n}\right)= \\
& =p_{t-1} \frac{\sum_{n} x_{t, n} w_{t-1, n}+\left(e_{t}-1\right) \sum_{n} x_{t, n} w_{t-1, n} x_{t-1, n}}{\sum_{n} x_{t-1, n} w_{t-1, n}-\sum_{n} x_{t, n} x_{t-1, n} w_{t-1, n}}
\end{aligned}
$$

where we have used (2.5) rewritten for time $t-1$ to get the second equality. Condition (2.12) is obtained imposing $p_{t}>0$, and the dynamics of the price return in (2.13) is immediately derived. From (2.6) rewritten for time $t+1$ it follows that

$$
w_{t+1, n}=w_{t, n}\left(1+x_{t, n}\left(r_{t+1}+e_{t+1}\right)\right) \quad \forall n \in\{1, \ldots, N\} \quad,
$$

which leads to (2.14). To obtain the wealth share dynamics, divide both sides of (A.1) by $w_{t+1}$ to have

$$
\begin{aligned}
\varphi_{t+1, n} & =\frac{w_{t, n}\left(1+x_{t, n}\left(r_{t+1}+e_{t+1}\right)\right)}{\sum_{m} w_{t+1, m}}=\frac{w_{t, n}\left(1+x_{t, n}\left(r_{t+1}+e_{t+1}\right)\right)}{\sum_{m} w_{t, m}+\left(r_{t+1}+e_{t+1}\right) \sum_{m} x_{t, m} w_{t, m}}= \\
& =\frac{\varphi_{t, n}\left(1+x_{t, n}\left(r_{t+1}+e_{t+1}\right)\right)}{1+\left(r_{t+1}+e_{t+1}\right) \sum_{m} x_{t, m} \varphi_{t, m}},
\end{aligned}
$$

where (A.1) has been used to get the second equality and we divided both numerator and denominator by the total wealth at time $t$ to get the third equality.

\section{B Proof of Proposition 2.2}

If (2.16) is valid for any $x$ it is also valid for their wealth-weighted average. Therefore one has

$$
\begin{aligned}
x_{\min } & \leq\left\langle x_{t}\right\rangle \\
x_{\min }^{2} & \leq x_{\max }, \\
x_{\min }\left(1-x_{\max }\right) & \leq\left\langle x_{t}\left(1-x_{t+1}\right)\right\rangle \leq x_{\max }^{2}, \\
& \leq x_{\max }\left(1-x_{\min }\right) .
\end{aligned}
$$


Since $e_{t+1}>0$ both factors in the left-hand side of (2.12) are positive and, therefore, this constraint is satisfied. At the same time, since the denominator of the expression in (2.13) is strictly greater than zero and the numerator is bounded, price return $r_{t}$ is bounded over time. Then, from (2.14) it follows that also agents' wealth returns $\rho_{t, n}$ are uniformly bounded over $n$ and $t$.

\section{Proof of Proposition 3.1}

Item $(i)$ is obvious after direct substitution of the equilibrium values into system (3.2). Item (ii) is equivalent to (2.12) rewritten at the equilibrium. Item (iii) follows from (2.14) and (3.3):

$$
\rho^{*}=x^{*}\left(r^{*}+\bar{e}\right)=l\left(r^{*}\right)\left(r^{*}+\bar{e}\right)=r^{*} .
$$

\section{Proof of Proposition 3.2}

The Jacobian matrix $J$ of the system at a fixed point reads

$$
J=\left\|\begin{array}{cc}
0 & f^{\prime} \\
-(1-\lambda) /\left(x^{*}\left(1-x^{*}\right)\right) & \lambda+(1-\lambda)\left(1+r^{*}\right) f^{\prime} /\left(x^{*}\left(1-x^{*}\right)\right)
\end{array}\right\|,
$$

and has the following characteristic polynomial

$$
\mu^{2}-\mu\left(\lambda+(1-\lambda) f^{\prime} \frac{1+r^{*}}{x^{*}\left(1-x^{*}\right)}\right)+(1-\lambda) f^{\prime} \frac{1}{x^{*}\left(1-x^{*}\right)} .
$$

It is well-known that both roots of this polynomial are inside the unit circle if the following three conditions are satisfied: $d<1, t<1+d$ and $t>-1-d$, where

$$
t=\lambda+(1-\lambda) f^{\prime} \frac{1+r^{*}}{x^{*}\left(1-x^{*}\right)} \quad \text { and } \quad d=(1-\lambda) f^{\prime} \frac{1}{x^{*}\left(1-x^{*}\right)} .
$$

The three inequalities (3.4) can now be obtained by direct substitution and taking into account that $l^{\prime}\left(r^{*}\right)=\left(x^{*}(1-\right.$ $\left.\left.x^{*}\right)\right) / r^{*}$.

\section{E Proof of Proposition 3.4}

The Jacobian matrix of the system at a fixed point reads

$$
\begin{array}{|ccc}
0 & f_{y}^{\prime} & f_{z}^{\prime} \\
-(1-\lambda) /\left(x^{*}\left(1-x^{*}\right)\right) & \lambda+(1-\lambda)\left(1+y^{*}\right) f_{y}^{\prime} /\left(x^{*}\left(1-x^{*}\right)\right) & (1-\lambda)\left(1+y^{*}\right) f_{z}^{\prime} /\left(x^{*}\left(1-x^{*}\right)\right) \\
0 & 0 & \lambda
\end{array}
$$

where $f_{z}^{\prime}$ is the derivative of $f$ with respect to the second variable computed at the equilibrium. One of the eigenvalues is $\lambda<1$, while the others are the roots of polynomial (D.1) with $f^{\prime}$ replaced by $f_{y}^{\prime}$. The statement immediately follows.

\section{F Proof of Proposition 4.1}

Since $\lambda_{n} \neq 1$ for all $n$, the first set of equalities in (4.5) follows immediately from block $\mathcal{Y}$. Then, the second part of (4.5) is also obvious from the equations in block $\mathcal{Z}$. Plugging the resulting relations into the equations of block $\mathcal{X}$ one has the third part of (4.5). Finally, relation (4.3) in equilibrium reads:

$$
r^{*}=\bar{e} \frac{\sum_{n=1}^{N-1} \varphi_{n}^{*} x_{n}^{* 2}+\left(1-\sum_{n=1}^{N-1} \varphi_{n}^{*}\right) x_{N}^{* 2}}{\sum_{n=1}^{N-1} \varphi_{n}^{*} x_{n}^{*}\left(1-x_{n}^{*}\right)+\left(1-\sum_{n=1}^{N-1} \varphi_{n}^{*}\right) x_{N}^{*}\left(1-x_{N}^{*}\right)} .
$$

Let us consider, first, the case when $r^{*}+\bar{e} \neq 0$ and derive the equilibria described in the first two items of the Proposition. From block $\mathcal{W}$ using (4.2) one obtains

$$
\varphi_{n}^{*}=0 \quad \text { or } \quad \sum_{m=1}^{N-1} \varphi_{m}^{*} x_{m}^{*}+\left(1-\sum_{m=1}^{N-1} \varphi_{m}^{*}\right) x_{N}^{*}=x_{n}^{*} \quad \forall n \in\{1, \ldots, N-1\} \quad .
$$


The set of equations (F.1) and (F.2) admits two types of solution, depending on how many equilibrium wealth shares are different from zero: either one or many.

To derive the first type of solution assume (4.6). In this case (F.2) is, obviously, automatically satisfied for all agents. From (F.1) one has $x_{1}^{*}=r^{*} /\left(\bar{e}+r^{*}\right)$ which together with the third part of (4.5) leads to (4.7).

To derive the second type of solution assume (4.8). In this case, the second equality of (F.2) must be satisfied for any $n \leq k$. Since its left-hand side does not depend on $n$, a $x_{1 \diamond k}^{*}$ must exist such that $x_{1}^{*}=\cdots=x_{k}^{*}=x_{1 \diamond k}^{*}$. Substituting $x_{n}^{*}=0$ for $n>k$ and $x_{n}^{*}=x_{1 \diamond k}^{*}$ for $n \leq k$ in (F.1) one gets $x_{1 \diamond k}^{*}=r^{*} /\left(\bar{e}+r^{*}\right)$. Combining this last relation with the third part of (4.5) for $n \leq k$ we get (4.9).

Considering, finally, the case when $r^{*}+\bar{e}=0$, we straight-forwardly derive the equilibria from the third item of the Proposition. Indeed, equations from block $\mathcal{W}$ are automatically satisfied, while (4.10) follows from (F.1).

The equilibrium wealth growth rate of the survivors is immediately obtained from the derived results and (2.14).

\section{G Proofs of Propositions in Section 4.3}

Before proving Propositions 4.2, 4.3 and 4.4 we need some preliminary results. The Jacobian matrix of the deterministic skeleton of system $(4.1)$ is a $(4 N-1) \times(4 N-1)$ matrix. Using the block structure introduced in Section 4.1 it can be separated into 16 blocks

$$
\boldsymbol{J}=\left\|\begin{array}{llll}
\frac{\partial \mathcal{X}}{\partial \mathcal{X}} & \frac{\partial \mathcal{X}}{\partial \mathcal{Y}} & \frac{\partial \mathcal{X}}{\partial \mathcal{Z}} & \frac{\partial \mathcal{X}}{\partial \mathcal{W}} \\
\frac{\partial \mathcal{Y}}{\partial \mathcal{X}} & \frac{\partial \mathcal{Y}}{\partial \mathcal{Y}} & \frac{\partial \mathcal{Y}}{\partial \mathcal{Z}} & \frac{\partial \mathcal{Y}}{\partial \mathcal{W}} \\
\frac{\partial \mathcal{Z}}{\partial \mathcal{X}} & \frac{\partial \mathcal{Z}}{\partial \mathcal{Y}} & \frac{\partial \mathcal{Z}}{\partial \mathcal{Z}} & \frac{\partial \mathcal{Z}}{\partial \mathcal{W}} \\
\frac{\partial \mathcal{W}}{\partial \mathcal{X}} & \frac{\partial \mathcal{W}}{\partial \mathcal{Y}} & \frac{\partial \mathcal{W}}{\partial \mathcal{Z}} & \frac{\partial \mathcal{W}}{\partial \mathcal{W}}
\end{array}\right\|
$$

The block $\partial \mathcal{X} / \partial \mathcal{X}$ is an $N \times N$ matrix containing the partial derivatives of the agents' present investment choices with respect to the agents' past investment choices. According to (2.8) the investment choice of any agent does not explicitly depend on the investment choices in the previous period, therefore,

$$
\left[\frac{\partial \mathcal{X}}{\partial \mathcal{X}}\right]_{n, m}=\frac{\partial f_{n}}{\partial x_{m}}=0, \quad 1 \leq n, m \leq N \quad,
$$

and this block is a zero matrix.

The block $\partial \mathcal{X} / \partial \mathcal{Y}$ is an $N \times N$ matrix containing the partial derivatives of the agents' investment choices with respect to the agents' return forecasts. Since the choice of any agent does not depend on the forecasts of other agents, this block is a diagonal matrix with diagonal elements

$$
\left[\frac{\partial \mathcal{X}}{\partial \mathcal{Y}}\right]_{n, n}=\frac{\partial f_{n}}{\partial y_{n}}=f_{n, y}^{\prime}, \quad 1 \leq n \leq N \quad .
$$

Analogously the $N \times N$ block $\partial \mathcal{X} / \partial \mathcal{Z}$ of the partial derivatives of the agents' investment choices with respect to the agents' variance forecasts is a diagonal matrix with diagonal elements

$$
\left[\frac{\partial \mathcal{X}}{\partial \mathcal{Z}}\right]_{n, n}=\frac{\partial f_{n}}{\partial z_{n}}=f_{n, z}^{\prime}, \quad 1 \leq n \leq N
$$

The block $\partial \mathcal{X} / \partial \mathcal{W}$ is an $N \times(N-1)$ matrix containing the partial derivatives of the agents' investment choices with respect to the agents' investment shares. Under Assumption 2 this is a zero matrix

$$
\left[\frac{\partial \mathcal{X}}{\partial \mathcal{W}}\right]_{n, m}=\frac{\partial f_{n}}{\partial \varphi_{m}}=0, \quad 1 \leq n \leq N, \quad 1 \leq m \leq N-1
$$

The definitions of the next blocks will make use of the function on the right-hand side of (4.3) which gives the evolution of the return. This function depends on the agents' previous investment choices $x_{t, n}$, the agents' wealth shares $\varphi_{t, n}$ and the agents' contemporaneous investment choices given by the investment functions $f_{n}$ for $n \in\{1, \ldots, N\}$. We denote the corresponding derivatives as $r_{x_{n}}^{\prime}, r_{\varphi_{n}}^{\prime}$ and $r_{f_{n}}^{\prime}$.

The block $\partial \mathcal{Y} / \partial \mathcal{X}$ is an $N \times N$ matrix containing the partial derivatives of the agents' return forecasts with respect to the agents' investment shares. The elements of this block read:

$$
\left[\frac{\partial \mathcal{Y}}{\partial \mathcal{X}}\right]_{n, m}=\frac{\partial y_{n}}{\partial x_{m}}=\left(1-\lambda_{n}\right) r_{x_{m}}^{\prime}, \quad 1 \leq n, m \leq N
$$


The block $\partial \mathcal{Y} / \partial \mathcal{Y}$ is an $N \times N$ matrix containing the partial derivatives of the agents' return forecasts with respect to the same set of variables. Using the chain rule one can easily check that this block contains the following elements:

$$
\left[\frac{\partial \mathcal{Y}}{\partial \mathcal{Y}}\right]_{n, m}=\frac{\partial y_{n}}{\partial y_{m}}=\lambda_{n} \delta_{n, m}+\left(1-\lambda_{n}\right) r_{f_{m}}^{\prime} f_{m, y}^{\prime}, \quad 1 \leq n, m \leq N
$$

where $\delta_{n, m}$ stands for the Kronecker delta.

The block $\partial \mathcal{Y} / \partial \mathcal{Z}$ is an $N \times N$ matrix containing the partial derivatives of the agents' return forecasts with respect to the agents' forecasts for variance. Using the chain rule we find that the elements of this block read:

$$
\left[\frac{\partial \mathcal{Y}}{\partial \mathcal{Z}}\right]_{n, m}=\frac{\partial y_{n}}{\partial z_{m}}=\left(1-\lambda_{n}\right) r_{f_{m}}^{\prime} f_{m, z}^{\prime}, \quad 1 \leq n, m \leq N
$$

The block $\partial \mathcal{Y} / \partial \mathcal{W}$ is an $N \times(N-1)$ matrix containing the partial derivatives of the agents' return forecasts with respect to the agents' wealth shares. The elements of this block are:

$$
\left[\frac{\partial \mathcal{Y}}{\partial \mathcal{W}}\right]_{n, m}=\frac{\partial y_{n}}{\partial \varphi_{m}}=\left(1-\lambda_{n}\right) r_{\varphi_{m}}^{\prime}, \quad 1 \leq n \leq N, \quad 1 \leq m \leq N-1
$$

The block $\partial \mathcal{Z} / \partial \mathcal{X}$ is an $N \times N$ matrix containing the partial derivatives of the agents' variance forecasts with respect to the agents' investment shares. In any equilibrium this block is a zero matrix:

$$
\left[\frac{\partial \mathcal{Z}}{\partial \mathcal{X}}\right]_{n, m}=\frac{\partial z_{n}}{\partial x_{m}}=0, \quad 1 \leq n, m \leq N
$$

Indeed, the derivative $\partial z_{n} / \partial x_{m}$ contains the factor $r_{t+1}-y_{t, n}$, which reduces to zero in any equilibrium due to (4.5).

Analogously, the block $\partial \mathcal{Z} / \partial \mathcal{Y}$ which is an $N \times N$ matrix containing the partial derivatives of the agents' variance forecasts with respect to the agents' return forecasts, is a zero block in equilibrium:

$$
\left[\frac{\partial \mathcal{Z}}{\partial \mathcal{Y}}\right]_{n, m}=\frac{\partial z_{n}}{\partial y_{m}}=0, \quad 1 \leq n, m \leq N .
$$

The same reasoning allows us to simplify the $N \times N$ block $\partial \mathcal{Z} / \partial \mathcal{Z}$ containing the partial derivatives of the agents' variance forecasts with respect to themselves. In equilibrium this is a diagonal matrix with diagonal elements

$$
\left[\frac{\partial \mathcal{Z}}{\partial \mathcal{Z}}\right]_{n, n}=\frac{\partial z_{n}}{\partial z_{n}}=\lambda_{n}, \quad 1 \leq n \leq N
$$

The block $\partial \mathcal{Z} / \partial \mathcal{W}$ is an $N \times(N-1)$ matrix containing the partial derivatives of the agents' variance forecasts with respect to the agents' wealth shares. Again, in any equilibrium this is a zero block:

$$
\left[\frac{\partial \mathcal{Z}}{\partial \mathcal{W}}\right]_{n, m}=\frac{\partial z_{n}}{\partial \varphi_{m}}=0, \quad 1 \leq n \leq N, \quad 1 \leq m \leq N-1
$$

The block $\partial \mathcal{W} / \partial \mathcal{X}$ is an $(N-1) \times N$ matrix containing the partial derivatives of the agents' wealth shares with respect to the agents' investment shares. It is:

$$
\left[\frac{\partial \mathcal{W}}{\partial \mathcal{X}}\right]_{n, m}=\frac{\partial \varphi_{n}}{\partial x_{m}}=\Phi_{n}^{x_{m}}+\Phi_{n}^{r} \cdot r_{x_{m}}^{\prime}, \quad 1 \leq n \leq N-1, \quad 1 \leq m \leq N
$$

where $\Phi_{n}^{x_{m}}=\partial \Phi_{n} / \partial x_{m}$ and $\Phi_{n}^{r}=\partial \Phi_{n} / \partial r$.

The block $\partial \mathcal{W} / \partial \mathcal{Y}$ is an $(N-1) \times N$ matrix containing the partial derivatives of the agents' wealth shares with respect to the agents' return forecast. It is

$$
\left[\frac{\partial \mathcal{W}}{\partial \mathcal{Y}}\right]_{n, m}=\frac{\partial \varphi_{n}}{\partial y_{m}}=\Phi_{n}^{r} \cdot r_{f_{m}}^{\prime} \cdot f_{m, y}^{\prime}, \quad 1 \leq n \leq N-1, \quad 1 \leq m \leq N
$$

The block $\partial \mathcal{W} / \partial \mathcal{Z}$ is an $(N-1) \times N$ matrix containing the partial derivatives of the agents' wealth shares with respect to the agents' variance forecasts. It is

$$
\left[\frac{\partial \mathcal{W}}{\partial \mathcal{Z}}\right]_{n, m}=\frac{\partial \varphi_{n}}{\partial z_{m}}=\Phi_{n}^{r} \cdot r_{f_{m}}^{\prime} \cdot f_{m, z}^{\prime}, \quad 1 \leq n \leq N-1, \quad 1 \leq m \leq N
$$


The block $\partial \mathcal{W} / \partial \mathcal{W}$ is an $(N-1) \times(N-1)$ matrix containing the partial derivatives of the agents' wealth shares with respect to the agents' wealth shares. The elements of this block are:

$$
\left[\frac{\partial \mathcal{W}}{\partial \mathcal{W}}\right]_{n, m}=\frac{\partial \varphi_{n}}{\partial \varphi_{m}}=\Phi_{n}^{\varphi_{m}}+\Phi_{n}^{r} \cdot r^{\varphi_{m}}, \quad 1 \leq n, m \leq N-1
$$

where $\Phi_{n}^{\varphi_{m}}=\partial \Phi_{n} / \partial \varphi_{m}$.

With the previous definitions one obtains the following

Lemma G.1. Let $\boldsymbol{x}^{*}$ be an equilibrium of the system (4.1) with $k \geq 1$ survivors. The Jacobian matrix computed at this point, $\boldsymbol{J}\left(\boldsymbol{x}^{*}\right)$, has the structure of the following matrix, where each one of the 16 blocks defined above is divided into 4 sub-blocks to display the upper-left $k \times k$ minor matrix. All the elements which are different from zero in all types of equilibria are denoted by the same symbol “ $\star$ ”, while for those elements which are non-zero only in the no-arbitrage equilibria we use symbol "

\begin{tabular}{|c|c|c|c|c|c|c|c|c|c|c|c|c|c|c|c|c|c|c|c|c|c|c|c|}
\hline 0 & $\ldots$ & 0 & 0 & $\ldots$ & 0 & $\star$ & $\ldots$ & 0 & 0 & $\ldots$ & 0 & $\star$ & $\ldots$ & 0 & 0 & $\ldots$ & 0 & 0 & $\ldots$ & 0 & 0 & $\ldots$ & 0 \\
\hline & $\ddots$ & $\vdots$ & $\vdots$ & $\ddots$ & $\vdots$ & . & $\ddots$. & $\vdots$ & : & $\ddots$ & $\vdots$ & & $\ddots$ & : & & $\ddots$ & $\vdots$ & $\vdots$ & $\ddots$ & : & $\vdots$ & $\ddots$. & $\vdots$ \\
\hline 0 & $\ldots$ & 0 & 0 & $\ldots$ & 0 & 0 & $\ldots$ & $\star$ & 0 & $\ldots$ & 0 & 0 & $\ldots$ & $\star$ & 0 & $\ldots$ & 0 & 0 & $\ldots$ & 0 & 0 & $\ldots$ & 0 \\
\hline 0 & $\ldots$ & 0 & 0 & $\ldots$ & 0 & 0 & $\ldots$ & 0 & $\star$ & $\ldots$ & 0 & 0 & $\ldots$ & 0 & $\star$ & $\ldots$ & 0 & 0 & $\ldots$ & 0 & 0 & $\ldots$ & 0 \\
\hline$\vdots$ & $\ddots$ & $\vdots$ & $\vdots$ & $\ddots$ & $\vdots$ & $\vdots$ & $\ddots$ & : & : & $\ddots$ & $\vdots$ & : & $\ddots$ & $\vdots$ & : & $\ddots$ & $\vdots$ & $\vdots$ & $\ddots$ & : & $\vdots$ & $\ddots$. & $\vdots$ \\
\hline 0 & $\ldots$ & 0 & 0 & $\ldots$ & 0 & 0 & $\ldots$ & 0 & 0 & $\ldots$ & $\star$ & 0 & $\ldots$ & 0 & 0 & $\ldots$ & $\star$ & 0 & $\ldots$ & 0 & 0 & $\ldots$ & 0 \\
\hline$\star$ & $\ldots$ & $\star$ & 0 & $\ldots$ & 0 & $\star$ & $\ldots$ & $\star$ & 0 & $\ldots$ & 0 & $\star$ & $\ldots$ & $\star$ & 0 & $\ldots$ & 0 & $\star$ & $\ldots$ & $\star$ & $\star$ & $\ldots$ & $\star$ \\
\hline$\vdots$ & $\ddots$ & $\vdots$ & $\vdots$ & $\ddots$ & . & : & $\ddots$. & $\vdots$ & . & $\ddots$ & $\vdots$ & . & $\ddots$ & & $\vdots$ & $\ddots$ & $\vdots$ & $\vdots$ & $\ddots$ & : & $\vdots$ & $\ddots$. & $\vdots$ \\
\hline$\star$ & $\ldots$ & $\star$ & 0 & $\ldots$ & 0 & $\star$ & $\ldots$ & $\star$ & 0 & $\ldots$ & 0 & $\star$ & $\ldots$ & $\star$ & 0 & $\ldots$ & 0 & $\star$ & $\ldots$ & $\star$ & $\star$ & $\ldots$ & $\star$ \\
\hline$\star$ & $\ldots$ & $\star$ & 0 & $\ldots$ & 0 & $\star$ & $\ldots$ & $\star$ & $\star$ & $\ldots$ & 0 & $\star$ & $\ldots$ & $\star$ & 0 & $\ldots$ & 0 & $\star$ & $\ldots$ & $\star$ & $\star$ & $\ldots$ & $\star$ \\
\hline : & $\ddots$ & $\vdots$ & $\vdots$ & $\ddots$ & $\vdots$ & $\vdots$ & $\ddots$. & • & • & $\ddots$ & $\cdot$ & . & $\ddots$ & $\vdots$ & : & $\ddots$ & $\vdots$ & $\vdots$ & $\ddots$ & . & $\vdots$ & $\ddots$ & $\vdots$ \\
\hline$\star$ & $\ldots$ & $\star$ & 0 & $\ldots$ & 0 & $\star$ & $\ldots$ & $\star$ & 0 & $\ldots$ & $\star$ & $\star$ & $\ldots$ & $\star$ & 0 & $\ldots$ & 0 & $\star$ & $\ldots$ & $\star$ & $\star$ & $\ldots$ & $\star$ \\
\hline 0 & $\ldots$ & 0 & 0 & $\ldots$ & 0 & 0 & $\ldots$ & 0 & 0 & $\ldots$ & 0 & $\star$ & $\ldots$ & 0 & 0 & $\ldots$ & 0 & 0 & $\ldots$ & 0 & 0 & $\ldots$ & 0 \\
\hline$\vdots$ & $\ddots$ & $\vdots$ & $\vdots$ & $\ddots$ & $\vdots$ & $\vdots$ & $\ddots$. & $\vdots$ & & $\ddots$ & : & & $\ddots$ & $\vdots$ & • & $\ddots$ & : & : & $\ddots$ & $\vdots$ & $\vdots$ & $\ddots$ & $\vdots$ \\
\hline 0 & $\ldots$ & 0 & 0 & $\ldots$ & 0 & 0 & $\ldots$ & 0 & 0 & $\ldots$ & 0 & 0 & $\ldots$ & $\star$ & 0 & $\ldots$ & 0 & 0 & $\ldots$ & 0 & 0 & $\ldots$ & 0 \\
\hline 0 & $\ldots$ & 0 & 0 & $\ldots$ & 0 & 0 & $\ldots$ & 0 & 0 & $\ldots$ & 0 & 0 & $\ldots$ & 0 & $\star$ & $\ldots$ & 0 & 0 & $\ldots$ & 0 & 0 & $\ldots$ & 0 \\
\hline$\vdots$ & $\ddots$ & $\vdots$ & : & $\ddots$ & $\vdots$ & $\vdots$ & $\ddots$ & $\vdots$ & : & $\ddots$ & $\vdots$ & : & $\ddots$ & $\vdots$ & : & $\ddots$ & : & : & $\ddots$ & $\vdots$ & $\vdots$ & $\ddots$ & $\vdots$ \\
\hline 0 & $\ldots$ & 0 & 0 & $\ldots$ & 0 & 0 & $\ldots$ & 0 & 0 & $\ldots$ & 0 & 0 & $\ldots$ & 0 & 0 & $\ldots$ & $\star$ & 0 & $\ldots$ & 0 & 0 & $\ldots$ & 0 \\
\hline$\star$ & $\ldots$ & $\star$ & 0 & $\ldots$ & 0 & $\square$ & $\ldots$ & $\square$ & 0 & $\ldots$ & 0 & $\square$ & $\ldots$ & $\square$ & 0 & $\ldots$ & 0 & $\star$ & $\ldots$ & $\star$ & $\star$ & $\ldots$ & $\star$ \\
\hline : & $\ddots$ & : & : & $\ddots$ & & : & 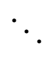 & : & : & $\ddots$ & $\vdots$ & $\cdot$ & $\cdot$ & $\vdots$ & $\vdots$ & $\ddots$ & $\vdots$ & $\vdots$ & $\cdot$ & $\vdots$ & $\vdots$ & $\ddots$ & $\vdots$ \\
\hline$\star$ & $\ldots$ & $\star$ & 0 & $\ldots$ & 0 & $\square$ & $\ldots$ & $\square$ & 0 & $\ldots$ & 0 & $\square$ & $\ldots$ & $\square$ & 0 & $\ldots$ & 0 & $\star$ & $\ldots$ & $\star$ & $\star$ & $\ldots$ & $\star$ \\
\hline 0 & $\ldots$ & 0 & 0 & $\ldots$ & 0 & 0 & $\ldots$ & 0 & 0 & $\ldots$ & 0 & 0 & $\ldots$ & 0 & 0 & $\ldots$ & 0 & 0 & $\ldots$ & 0 & $\star$ & $\ldots$ & 0 \\
\hline : & $\ddots$ & $\vdots$ & : & $\ddots$ & $\cdot$ & $\vdots$ & $\ddots$ & . & : & $\ddots$ & $\vdots$ & . & $\ddots$ & $\vdots$ & $\vdots$ & $\ddots$ & : & : & $\ddots$ & : & $\vdots$ & $\ddots$ & $\vdots$ \\
\hline 0 & $\ldots$ & 0 & 0 & $\ldots$ & 0 & 0 & $\ldots$ & 0 & 0 & $\ldots$ & 0 & 0 & $\ldots$ & 0 & 0 & $\ldots$ & 0 & 0 & $\ldots$ & 0 & 0 & $\ldots$ & $\star$ \\
\hline
\end{tabular}

Proof. Let us consider, first, those equilibria where $r^{*} \neq-\bar{e}$. For all $n, l \in\{1, \ldots, N-1\}$ and $m \in\{1, \ldots, N\}$ one has

$$
\begin{aligned}
\Phi_{n}^{r} & =\varphi_{n}^{*} \frac{x_{n}^{*}-x_{1 \diamond k}^{*}}{1+r^{*}}=0, \quad \Phi_{n}^{x_{m}}=\varphi_{n}^{*}\left(\delta_{n, m}-\varphi_{m}^{*}\right) \frac{\bar{e}+r^{*}}{1+r^{*}}, \\
\Phi_{n}^{\varphi_{l}} & =\frac{\delta_{n, l}\left(1+x_{n}^{*}\left(r^{*}+\bar{e}\right)\right)-\varphi_{n}^{*}\left(r^{*}+\bar{e}\right)\left(x_{l}^{*}-x_{N}^{*}\right)}{1+r^{*}} .
\end{aligned}
$$

Therefore, blocks $[\partial \mathcal{W} / \partial \mathcal{Y}]$ and $[\partial \mathcal{W} / \partial \mathcal{Z}]$ are zero matrices, while the following blocks can be simplified:

$$
\left[\frac{\partial \mathcal{W}}{\partial \mathcal{X}}\right]_{n, m}=\left\{\begin{array}{ll}
\Phi_{n}^{x_{m}} & m, n \leq k \\
0 & \text { otherwise }
\end{array} \text { and } \quad\left[\frac{\partial \mathcal{W}}{\partial \mathcal{W}}\right]_{n, m}= \begin{cases}0 & n>k, \quad n \neq m \\
\Phi_{n}^{\varphi_{m}} & \text { otherwise }\end{cases}\right.
$$

Furthermore, for $m \in\{1, \ldots, N\}$ it is

$$
r_{x_{m}}^{\prime}=-\varphi_{m}^{*} \frac{1}{x_{1 \diamond k}^{*}\left(1-x_{1 \diamond k}^{*}\right)}, \quad r_{f_{m}}^{\prime}=\varphi_{m}^{*} \frac{1+r^{*}}{x_{1 \diamond k}^{*}\left(1-x_{1 \diamond k}^{*}\right)}
$$


Thus $r_{x_{m}}^{\prime}=r_{f_{m}}^{\prime}=0$ for $m>k$, and the structure above (with zeros instead of

immediately follows. Moreover,

$$
r_{\varphi_{m}}^{\prime}=\frac{r^{*}+\bar{e}}{x_{1 \diamond k}^{*}\left(1-x_{1 \diamond k}^{*}\right)}\left(x_{m}^{*}\left(x_{m}^{*}-x_{1 \diamond k}^{*}\right)-x_{N}^{*}\left(x_{N}^{*}-x_{1 \diamond k}^{*}\right)\right) \quad, \quad \forall m \in\{1, \ldots, N-1\} \quad .
$$

Second, we consider no-arbitrage equilibria. For $n, l \in\{1, \ldots, N-1\}$ and $m \in\{1, \ldots, N\}$ one has

$$
\begin{aligned}
& \Phi_{n}^{x_{m}}=0, \quad \Phi_{n}^{\varphi_{l}}=\delta_{n, l}, \quad \Phi_{n}^{r}=\varphi_{n}^{*} x_{n}^{*}, \\
& r_{x_{m}}^{\prime}=\varphi_{m}^{*} \frac{1-\bar{e}}{\left\langle x^{2}\right\rangle}, \quad r_{f_{m}}^{\prime}=-\varphi_{m}^{*} \frac{1}{\left\langle x^{2}\right\rangle}, \quad r_{\varphi_{n}}^{\prime}=-\bar{e} \frac{x_{n}^{*}-x_{N}^{*}}{\left\langle x^{2}\right\rangle} .
\end{aligned}
$$

Therefore

$$
\begin{aligned}
& {\left[\frac{\partial \mathcal{W}}{\partial \mathcal{X}}\right]_{n, m}=\left\{\begin{array}{ll}
\Phi_{n}^{r} r_{x_{m}}^{\prime} & m, n \leq k \\
0 & \text { otherwise }
\end{array}, \quad\left[\begin{array}{l}
\left.\frac{\partial \mathcal{W}}{\partial \mathcal{Y}}\right]_{n, m} \\
0
\end{array}= \begin{cases}\Phi_{n}^{r} r_{f_{m}}^{\prime} f_{m, y}^{\prime} & m, n \leq k \\
0 & \text { otherwise }\end{cases} \right.\right.} \\
& {\left[\frac{\partial \mathcal{W}}{\partial \mathcal{Z}}\right]_{n, m}=\left\{\begin{array}{ll}
\Phi_{n}^{r} r_{f_{m}}^{\prime} f_{m, z}^{\prime} & m, n \leq k \\
0 & \text { otherwise }
\end{array} \quad, \quad\left[\frac{\partial \mathcal{W}}{\partial \mathcal{W}}\right]_{n, m}= \begin{cases}\delta_{n, m}+\Phi_{n}^{r} r_{\varphi_{m}}^{\prime} & n \leq k \\
\delta_{n, m} & \text { otherwise }\end{cases} \right.}
\end{aligned}
$$

Lemma G.2. Consider equilibrium $\boldsymbol{x}^{*}$ with $r^{*} \neq-\bar{e}$. The characteristic polynomial $P_{J}$ of the matrix $\boldsymbol{J}\left(\boldsymbol{x}^{*}\right)$ reads

$$
\begin{aligned}
P_{J}(\mu)=(-1)^{N} \mu^{N-1}(1-\mu)^{k-1} \prod_{j=k+1}^{N}\left(\frac{1+x_{j}^{*}\left(r^{*}+\bar{e}\right)}{1+r^{*}}-\mu\right) \prod_{j=1}^{k}\left(\lambda_{j}-\mu\right) \prod_{j=k+1}^{N}\left(\lambda_{j}-\mu\right)^{2} \\
\left(\mu \prod_{j=1}^{k}\left(\lambda_{j}-\mu\right)+\frac{\left(1+r^{*}\right) \mu-1}{x_{1 \diamond k}^{*}\left(1-x_{1 \diamond k}^{*}\right)} \sum_{j=1}^{k}\left(\varphi_{j}^{*} f_{j, y}^{\prime}\left(1-\lambda_{j}\right) \prod_{i=1, i \neq j}^{k}\left(\lambda_{i}-\mu\right)\right)\right)
\end{aligned}
$$

Proof. The following proof is constructive: we will identify in succession the factors appearing in (G.6). At each step, a set of eigenvalues is found and the problem is reduced to the analysis of the residual matrix obtained removing the rows and columns associated with the relative eigenspace.

Consider the Jacobian matrix in Lemma G.1. One can easily see that in each of the $N$ rows belonging to the third row of blocks (blocks are separated by single lines) the only non-zero entries are the $\lambda$ 's on the diagonal of $[\partial \mathcal{Z} / \partial \mathcal{Z}]$. Consequently, $\lambda_{1}, \ldots, \lambda_{N}$ are eigenvalues of the matrix, with multiplicity (at least) one. Also in each of the last $N-1-k$ rows of the matrix, the only non-zero entries belong to the main diagonal of $[\partial \mathcal{W} / \partial \mathcal{W}]$. Thus, when $k<N-1$, we have the $N-1-k$ eigenvalues $\Phi_{n}^{\varphi_{n}}$ for $k+1 \leq n \leq N-1$, computed in (G.2). It is also obvious that the last $N-k$ columns of the leftmost block of column contain only zero entries so that the matrix possesses eigenvalue 0 with (at least) multiplicity $N-k$. A first contribution to the characteristic polynomial is, therefore, determined as

$$
(-\mu)^{N-k} \prod_{j=1}^{N}\left(\lambda_{j}-\mu\right) \prod_{j=k+1}^{N-1}\left(\Phi_{j}^{\varphi_{j}}-\mu\right)=(-\mu)^{N-k} \prod_{j=1}^{N}\left(\lambda_{j}-\mu\right) \prod_{j=k+1}^{N-1}\left(\frac{1+x_{j}^{*}\left(r^{*}+\bar{e}\right)}{1+r^{*}}-\mu\right)
$$

In order to find the remaining factors we eliminate the rows and columns associated with the previous eigenvalues. Consider now the last $N-k$ columns in the second block of columns in the remaining matrix. The only non zero elements are on the main diagonal of $[\partial \mathcal{Y} / \partial \mathcal{Y}]$. If $k<N$, this leads to a second contribution

$$
\prod_{j=k+1}^{N}\left(\lambda_{j}-\mu\right)
$$

After the corresponding further elimination of rows and columns the following matrix is obtained

\begin{tabular}{ccc|ccc|ccc}
0 & $\cdots$ & 0 & $f_{1, y}^{\prime}$ & $\cdots$ & 0 & 0 & $\cdots$ & 0 \\
$\vdots$ & $\ddots$ & $\vdots$ & $\vdots$ & $\ddots$ & $\vdots$ & $\vdots$ & $\ddots$ & $\vdots$ \\
0 & $\cdots$ & 0 & 0 & $\cdots$ & $f_{k, y}^{\prime}$ & 0 & $\cdots$ & 0 \\
\hline$\left(1-\lambda_{1}\right) r_{x_{1}}^{\prime}$ & $\cdots$ & $\left(1-\lambda_{1}\right) r_{x_{k}}^{\prime}$ & $\lambda_{1}+\left(1-\lambda_{1}\right) r_{f_{1}}^{\prime} f_{1, y}^{\prime}$ & $\cdots$ & $\left(1-\lambda_{1}\right) r_{f_{k}}^{\prime} f_{k, y}^{\prime}$ & $\left(1-\lambda_{1}\right) r_{\varphi_{1}}^{\prime}$ & $\cdots$ & $\left(1-\lambda_{1}\right) r_{\varphi_{k}}^{\prime}$ \\
$\vdots$ & $\ddots$ & $\vdots$ & $\vdots$ & $\ddots$ & $\vdots$ & $\ddots$ & $\vdots$ \\
$\left(1-\lambda_{k}\right) r_{x_{1}}^{\prime}$ & $\cdots$ & $\left(1-\lambda_{k}\right) r_{x_{k}}^{\prime}$ & $\left(1-\lambda_{k}\right) r_{f_{1}}^{\prime} f_{1, y}^{\prime}$ & $\cdots$ & $\lambda_{k}+\left(1-\lambda_{k}\right) r_{f_{k}}^{\prime} f_{k, y}^{\prime}$ & $\left(1-\lambda_{k}\right) r_{\varphi_{1}}^{\prime}$ & $\cdots$ & $\left(1-\lambda_{k}\right) r_{\varphi_{k}}^{\prime}$ \\
\hline$\Phi_{1}^{x_{1}}$ & $\cdots$ & $\Phi_{1}^{x} k$ & 0 & $\cdots$ & 0 & $\Phi_{1}^{\varphi_{1}}$ & $\cdots$ & $\Phi_{1}^{\varphi_{k}}$ \\
$\vdots$ & $\ddots$ & $\vdots$ & $\vdots$ & $\ddots$ & $\vdots$ & $\vdots$ & $\ddots$ & $\vdots$ \\
$\Phi_{k}^{x_{1}}$ & $\cdots$ & $\Phi_{k}^{x_{k}}$ & 0 & $\cdots$ & 0 & $\Phi_{k}^{\varphi_{1}}$ & $\cdots$ & $\Phi_{k}^{\varphi_{k}}$
\end{tabular}


This matrix, which we call $\boldsymbol{L}$, has dimension $3 k \times 3 k$ when $k<N$. If $k=N$, representation (G.9) is, strictly speaking, not correct, since there are only $N-1$ wealth shares $\varphi$ 's. In this case, the correct matrix has dimension $(3 N-1) \times(3 N-1)$ and can be obtained from (G.9) through elimination the last row and the last column. We will compute now the characteristic polynomial, i.e. the $\operatorname{determinant} \operatorname{det}(\boldsymbol{L}-\mu \boldsymbol{I})$, where $\boldsymbol{I}$ denotes an identity matrix of the corresponding dimension. We consider separately the following two cases: when $k<N$ and when $k=N$.

If $k<N$, then from (G.2) it follows that for $n, m \leq k$ one has

$$
\Phi_{n}^{\varphi_{m}}=\left\{\begin{array}{ll}
1-\varphi_{n}^{*} v & \text { if } n=m \\
-\varphi_{n}^{*} v & \text { otherwise }
\end{array}, \quad \text { where } \quad v=\left(x_{1 \diamond k}^{*}-x_{N}^{*}\right) \frac{\bar{e}+r^{*}}{1+r^{*}} .\right.
$$

Moreover, since all survivors invest share $x_{1 \diamond k}$, it follows from (G.4) that for $m \leq k$

$$
r_{\varphi_{m}}^{\prime}=v b, \quad \text { where } \quad b=x_{N}^{*} \frac{1+r^{*}}{x_{1 \diamond k}^{*}\left(1-x_{1 \diamond k}^{*}\right)} .
$$

Therefore, the last block of columns in the matrix $\boldsymbol{L}-\lambda \boldsymbol{I}$ can be rewritten as

$$
\left\|v \boldsymbol{b}+\boldsymbol{b}_{1}|\ldots| v \boldsymbol{b}+\boldsymbol{b}_{k}\right\|
$$

where we introduce the following column vectors

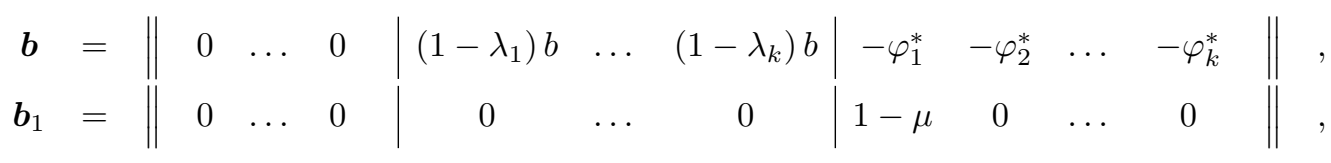

$$
\begin{aligned}
& \boldsymbol{b}_{k}=\| \begin{array}{lll|llll|llll}
0 & \ldots & 0 & 0 & \ldots & 0 & 0 & 0 & \ldots & 1-\mu
\end{array} .
\end{aligned}
$$

Based on the above representation of the last block, we apply the multilinear property to the $\operatorname{determinant} \operatorname{det}(\boldsymbol{L}-\lambda \boldsymbol{I})$. Thus, we consider each of the last columns as a sum of two terms and end up with a sum of $2^{k}$ determinants. Notice, however, that many of them are zeros, since they contain two or more columns proportional to vector $\boldsymbol{b}$. There are only $k+1$ non-zero elements in the expansion. One of them has the following structure of the last block of columns:

$$
\left\|\boldsymbol{b}_{1}|\ldots \ldots+\ldots| \boldsymbol{b}_{k}\right\|
$$

while all the other non-zero terms have column $v \boldsymbol{b}$ instead of $\boldsymbol{b}_{\nu}$ (for all $\nu \in\{1, \ldots, k\}$ ) on the $\nu$ 'th place of the last block of columns:

$$
\left\|\boldsymbol{b}_{1}|\ldots| v \boldsymbol{b}|\ldots| \boldsymbol{b}_{k}\right\|
$$

The matrix with the former block contains a diagonal lower-right corner. Its determinant is equal to $(1-\mu)^{k} \operatorname{det} \boldsymbol{M}$, with

$$
\boldsymbol{M}=\left\|\begin{array}{ccc|ccc}
-\mu & \ldots & 0 & f_{1, y}^{\prime} & \ldots & 0 \\
\vdots & \ddots & \vdots & \vdots & \ddots & \vdots \\
0 & \ldots & -\mu & 0 & \ldots & f_{k, y}^{\prime} \\
\hline\left(1-\lambda_{1}\right) r_{x_{1}}^{\prime} & \ldots & \left(1-\lambda_{1}\right) r_{x_{k}}^{\prime} & \lambda_{1}-\mu+\left(1-\lambda_{1}\right) r_{f_{1}}^{\prime} f_{1, y}^{\prime} & \ldots & \left(1-\lambda_{1}\right) r_{f_{k}}^{\prime} f_{k, y}^{\prime} \\
\vdots & \ddots & \vdots & \vdots & \ddots & \vdots \\
\left(1-\lambda_{k}\right) r_{x_{1}}^{\prime} & \ldots & \left(1-\lambda_{k}\right) r_{x_{k}}^{\prime} & \left(1-\lambda_{k}\right) r_{f_{1}}^{\prime} f_{1, y}^{\prime} & \ldots & \lambda_{k}-\mu+\left(1-\lambda_{k}\right) r_{f_{k}}^{\prime} f_{k, y}^{\prime}
\end{array}\right\|
$$

The remaining $k$ determinants can be simplified in an analogous way, so that

$$
\operatorname{det}(\boldsymbol{L}-\lambda \boldsymbol{I})=(1-\mu)^{k} \operatorname{det} \boldsymbol{M}+(1-\mu)^{k-1} \sum_{\nu=1}^{k} \operatorname{det} \boldsymbol{M}_{\nu}
$$


where for $\nu \in\{1, \ldots, k\}$ we introduce the following matrix with constants $v$ and $b$ defined in (G.10) and (G.11)

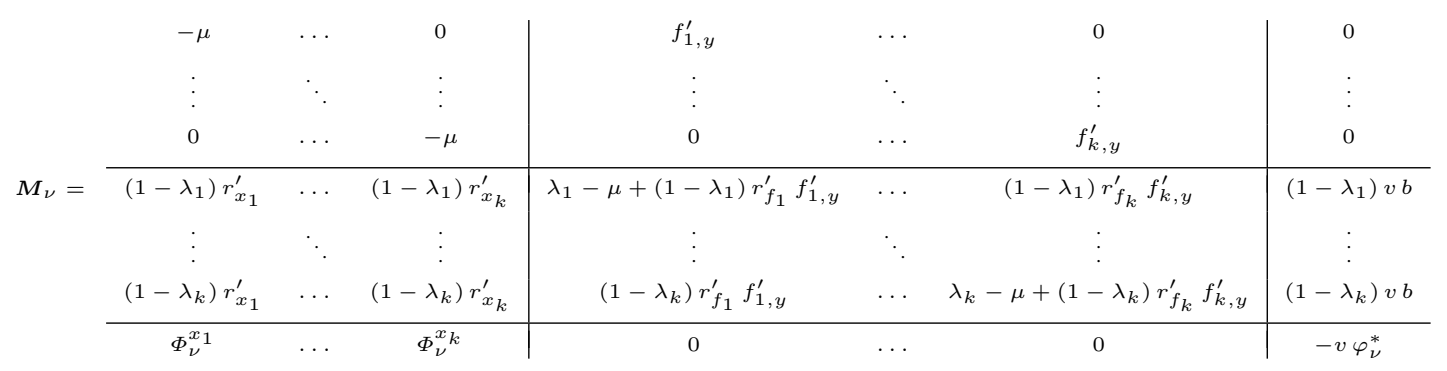

Our next step consists in the computation of the determinants of matrices $M$ and $M_{\nu}$. We will again exploit the multilinear property of the determinant. To compute the first determinant we rewrite the matrix as follows

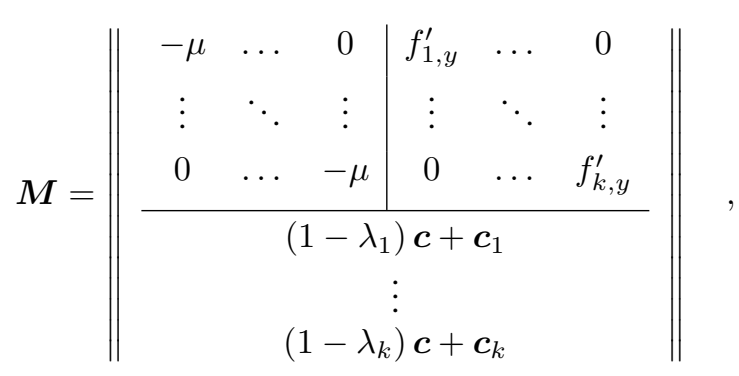

where the following $k+1$ row vectors were introduced

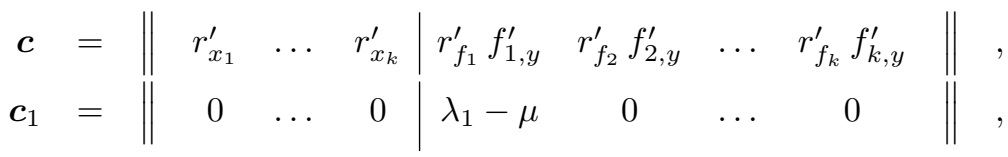

$$
\begin{aligned}
& \boldsymbol{c}_{k}=\| \begin{array}{lll|llll}
0 & \ldots & 0 & 0 & 0 & \ldots & \lambda_{k}-\mu
\end{array}
\end{aligned}
$$

Applying the multilinear property to the last $k$ rows in the determinant in (G.13), we get a sum of $2^{k}$ determinants. Many of them are zero, since they contain two or more rows proportional to the vector $c$. One of the remaining determinants belongs to the lower-diagonal matrix with vectors $\left\{\boldsymbol{c}_{1}, \ldots, \boldsymbol{c}_{k}\right\}$ in the last $k$ rows. All other non-zero determinants come from $k$ different matrices which can be obtained from this lower-diagonal matrix by substitution of one of the rows by vector $\boldsymbol{c}$. All these determinants can be easily computed, so that we have the following

$$
\operatorname{det} \boldsymbol{M}=(-\mu)^{k} \prod_{j=1}^{k}\left(\lambda_{j}-\mu\right)+\sum_{j=1}^{k}\left((-\mu)^{k-1}\left(1-\lambda_{j}\right) f_{j, y}^{\prime}\left(-\mu r_{f_{j}}^{\prime}-r_{x_{j}}^{\prime}\right) \prod_{i=1, i \neq j}^{k}\left(\lambda_{i}-\mu\right)\right) .
$$

Analogously for all $\nu \in\{1, \ldots, k\}$ we get

$$
\operatorname{det} \boldsymbol{M}_{\nu}=\left(-v \varphi_{\nu}^{*}\right)(-\mu)^{k} \prod_{j=1}^{k}\left(\lambda_{j}-\mu\right)+\sum_{j=1}^{k}\left((-\mu)^{k-1}\left(1-\lambda_{j}\right) f_{j, y}^{\prime} v \cdot \operatorname{det} \boldsymbol{M}_{\nu, j} \cdot \prod_{i=1, i \neq j}^{k}\left(\lambda_{i}-\mu\right)\right)
$$

where $M_{\nu, j}$ is the following $3 \times 3$ matrix

$$
\boldsymbol{M}_{\nu, j}=\left\|\begin{array}{ccc}
-\mu & 1 & 0 \\
r_{x_{j}}^{\prime} & r_{f_{j}}^{\prime} & b \\
\Phi_{\nu}^{x_{j}} & 0 & -\varphi_{\nu}^{*}
\end{array}\right\| \quad \text { with } \quad \operatorname{det} \boldsymbol{M}_{\nu, j}=b \Phi_{\nu}^{x_{j}}+\varphi_{\nu}^{*}\left(\mu r_{f_{j}}^{\prime}+r_{x_{j}}^{\prime}\right)
$$


Plugging the values of derivatives $r_{f_{j}}^{\prime}$ and $r_{x_{j}}^{\prime}$ computed in (G.3) into the corresponding expressions, we get the following

$$
\begin{aligned}
\sum_{\nu=1}^{k} \operatorname{det} \boldsymbol{M}_{\nu} & =-v(-\mu)^{k} \prod_{j=1}^{k}\left(\lambda_{j}-\mu\right)+v \sum_{j=1}^{k}\left((-\mu)^{k-1}\left(1-\lambda_{j}\right) f_{j, y}^{\prime} \prod_{i=1, i \neq j}^{k}\left(\lambda_{i}-\mu\right) \cdot \sum_{\nu=1}^{k} \operatorname{det} \boldsymbol{M}_{\nu, j}\right)= \\
& =-v(-\mu)^{k} \prod_{j=1}^{k}\left(\lambda_{j}-\mu\right)+v \sum_{j=1}^{k}\left((-\mu)^{k-1}\left(1-\lambda_{j}\right) f_{j, y}^{\prime} \prod_{i=1, i \neq j}^{k}\left(\lambda_{i}-\mu\right) \cdot\left(\mu r_{f_{j}}^{\prime}+r_{x_{j}}^{\prime}\right)\right)= \\
& =-v \operatorname{det} \boldsymbol{M}
\end{aligned}
$$

where to get the second equality we observe that $\sum_{\nu=1}^{k} \Phi_{\nu}^{x_{j}}=0$, which can be easily verified from (G.2), while the last equality follows from comparison with (G.14).

Now we are in the position to finish the computation of the $\operatorname{det}(\boldsymbol{L}-\lambda \boldsymbol{I})$ started in (G.12). It reads

$$
\operatorname{det}(\boldsymbol{L}-\lambda \boldsymbol{I})=(1-\mu-v)(1-\mu)^{k-1} \operatorname{det} \boldsymbol{M}=\left(\frac{1+x_{N}^{*}\left(r^{*}+\bar{e}\right)}{1+r^{*}}-\mu\right)(1-\mu)^{k-1} \operatorname{det} \boldsymbol{M} .
$$

If $k=N$, i.e. all agents survive, then all investment shares are the same. In this case, according to (G.2) and (G.4), all elements in the last block of columns of matrix (G.9) are zeros apart from the ones on the diagonal in the lowest $(N-1) \times(N-1)$ matrix. It contributes to the characteristic polynomial by the factor $(1-\mu)^{N-1}$. The remaining part is the determinant of matrix $M$ in this case. This is consistent with (G.15).

Bringing now together the contributions in (G.7), (G.8) and (G.15), and also computing determinant of matrix $M$ in (G.14) in equilibrium, we, finally, get the characteristic polynomial (G.6).

Lemma G.3. Consider no-arbitrage equilibrium $\boldsymbol{x}^{*}$. The characteristic polynomial $P_{J}$ of the matrix $\boldsymbol{J}\left(\boldsymbol{x}^{*}\right)$ reads

$$
\begin{aligned}
& P_{J}(\mu)=(-1)^{N-1} \mu^{N-1}(1-\mu)^{N-2}(\mu-1+\bar{e}) \prod_{j=1}^{k}\left(\lambda_{j}-\mu\right) \prod_{j=k+1}^{N}\left(\lambda_{j}-\mu\right)^{2} \\
&\left(\mu \prod_{j=1}^{k}\left(\lambda_{j}-\mu\right)+\frac{1-\mu}{\left\langle x^{2}\right\rangle} \sum_{j=1}^{k}\left(\varphi_{j}^{*} f_{j, y}^{\prime}\left(1-\lambda_{j}\right) \prod_{i=1, i \neq j}^{k}\left(\lambda_{i}-\mu\right)\right)\right)
\end{aligned}
$$

Proof. Since the proof below is analogous to the proof of Lemma G.2, some details are omitted. In particular, we confine analysis on the case when $k<N$. From the Jacobian matrix in Lemma G.1 it follows that (i) in each of the $N$ rows belonging to the third row of blocks the only non-zero entries are the $\lambda$ 's on the diagonal of $[\partial \mathcal{Z} / \partial \mathcal{Z}]$, (ii) in each of the last $N-1-k$ rows of the matrix, the only non-zero entries belong to the main diagonal of $[\partial \mathcal{W} / \partial \mathcal{W}]$ and equal to 1 , (iii) the last $N-k$ columns of the leftmost blocks contain only zero entries. Together, it gives the first entry in the characteristic polynomial:

$$
(-\mu)^{N-k}(1-\mu)^{N-1-k} \prod_{j=1}^{N}\left(\lambda_{j}-\mu\right) .
$$

Consider the last $N-k$ columns in the second block of columns of the matrix obtained after the corresponding elimination of the rows and columns. The only non zero elements are on the main diagonal of $[\partial \mathcal{Y} / \partial \mathcal{Y}]$. This leads to a second contribution

$$
\prod_{j=k+1}^{N}\left(\lambda_{j}-\mu\right)
$$


After further elimination of rows and columns, the following matrix is obtained

\begin{tabular}{ccc|ccc|ccc}
0 & $\cdots$ & 0 & $f_{1, y}^{\prime}$ & $\cdots$ & 0 & 0 & $\cdots$ & 0 \\
$\vdots$ & $\ddots$ & $\vdots$ & $\vdots$ & $\ddots$ & $\vdots$ & $\vdots$ & $\ddots$ & $\vdots$ \\
0 & $\cdots$ & 0 & 0 & $\cdots$ & $f_{k, y}^{\prime}$ & 0 & $\cdots$ & 0 \\
\hline$\left(1-\lambda_{1}\right) r_{x_{1}}^{\prime}$ & $\cdots$ & $\left(1-\lambda_{1}\right) r_{x_{k}}^{\prime}$ & $\lambda_{1}+\left(1-\lambda_{1}\right) r_{f_{1}}^{\prime} f_{1, y}^{\prime}$ & $\cdots$ & $\left(1-\lambda_{1}\right) r_{f_{k}}^{\prime} f_{k, y}^{\prime}$ & $\left(1-\lambda_{1}\right) r_{\varphi_{1}}^{\prime}$ & $\cdots$ & $\left(1-\lambda_{1}\right) r_{\varphi_{k}}^{\prime}$ \\
$\vdots$ & $\ddots$ & $\vdots$ & $\vdots$ & $\ddots$ & $\vdots$ & $\ddots$ & $\vdots$ \\
$\left(1-\lambda_{k}\right) r_{x_{1}}^{\prime}$ & $\cdots$ & $\left(1-\lambda_{k}\right) r_{x_{k}}^{\prime}$ & $\left(1-\lambda_{k}\right) r_{f_{1}}^{\prime} f_{1, y}^{\prime}$ & $\cdots$ & $\lambda_{k}+\left(1-\lambda_{k}\right) r_{f_{k}}^{\prime} f_{k, y}^{\prime}$ & $\left(1-\lambda_{k}\right) r_{\varphi_{1}}^{\prime}$ & $\cdots$ & $\left(1-\lambda_{k}\right) r_{\varphi_{k}}^{\prime}$ \\
\hline$\Phi_{1}^{r} r_{x_{1}}^{\prime}$ & $\cdots$ & $\Phi_{1}^{r} r_{x_{k}}^{\prime}$ & $\Phi_{1}^{r} r_{f_{1}}^{\prime} f_{1, y}^{\prime}$ & $\cdots$ & $\Phi_{1}^{r} r_{f_{k}}^{\prime} f_{k, y}^{\prime}$ & $1+\Phi_{1}^{r} r_{\varphi_{1}}^{\prime}$ & $\cdots$ & $\Phi_{1}^{r} r_{\varphi_{k}}^{\prime}$ \\
$\vdots$ & $\ddots$ & $\vdots$ & $\vdots$ & $\ddots$ & $\vdots$ & $\vdots$ & $\ddots$ & $\vdots$ \\
$\Phi_{k}^{r} r_{x_{1}}^{\prime}$ & $\cdots$ & $\Phi_{k}^{r} r_{x_{k}}^{\prime}$ & $\Phi_{k}^{r} r_{f_{1}}^{\prime} f_{1, y}^{\prime}$ & $\cdots$ & $\Phi_{k}^{r} r_{f_{k}}^{\prime} f_{k, y}^{\prime}$ & $\Phi_{k}^{r} r_{\varphi_{1}}^{\prime}$ & $\cdots$ & $1+\Phi_{k}^{r} r_{\varphi_{k}}^{\prime}$
\end{tabular}

We denote this matrix as $\boldsymbol{L}$ and compute the remaining term in the characteristic polynomial of the original system as $\operatorname{det}(\boldsymbol{L}-\mu \boldsymbol{I})$, where $\boldsymbol{I}$ is an identity $3 k \times 3 k$ matrix. Applying the multilinear property of the determinant to the last block of columns we get

$$
\operatorname{det}(\boldsymbol{L}-\mu \boldsymbol{I})=(1-\mu)^{k} \operatorname{det} \boldsymbol{M}+(1-\mu)^{k-1} \operatorname{det} \boldsymbol{N}_{\nu},
$$

where matrix $M$ was defined in (G.13) (recall, however that the partial derivatives $r_{x_{j}}^{\prime}$ and $r_{f_{j}}^{\prime}$ have now other values), and for all $\nu \in\{1, \ldots, k\}$ we introduce matrix

$$
\boldsymbol{N}_{\nu}=\begin{array}{ccc|ccc|c}
-\mu & \ldots & 0 & f_{1, y}^{\prime} & \cdots & 0 & 0 \\
\vdots & \ddots & \vdots & \vdots & \ddots & \vdots & \vdots \\
0 & \cdots & -\mu & 0 & \ldots & f_{k, y}^{\prime} & 0 \\
\hline\left(1-\lambda_{1}\right) r_{x_{1}}^{\prime} & \cdots & \left(1-\lambda_{1}\right) r_{x_{k}}^{\prime} & \lambda_{1}-\mu+\left(1-\lambda_{1}\right) r_{f_{1}}^{\prime} f_{1, y}^{\prime} & \cdots & \left(1-\lambda_{1}\right) r_{f_{k}}^{\prime} f_{k, y}^{\prime} & \left(1-\lambda_{1}\right) r_{\varphi_{\nu}}^{\prime} \\
\vdots & \ddots & \vdots & \vdots & \ddots & \vdots \\
\left(1-\lambda_{k}\right) r_{x_{1}}^{\prime} & \cdots & \left(1-\lambda_{k}\right) r_{x_{k}}^{\prime} & \left(1-\lambda_{k}\right) r_{f_{1}}^{\prime} f_{1, y}^{\prime} & \cdots & \lambda_{k}-\mu+\left(1-\lambda_{k}\right) r_{f_{k}}^{\prime} f_{k, y}^{\prime} & \left(1-\lambda_{k}\right) r_{\varphi_{\nu}}^{\prime} \\
\hline \Phi_{\nu}^{r} r_{x_{1}}^{\prime} & \cdots & \Phi_{\nu}^{r} r_{x_{k}}^{\prime} & \Phi_{\nu}^{r} r_{f_{1}}^{\prime} f_{1, y}^{\prime} & \cdots & \Phi_{\nu}^{r} r_{f_{k}}^{\prime} f_{k, y}^{\prime} & \Phi_{\nu}^{r} r_{\varphi_{\nu}}^{\prime}
\end{array}
$$

To compute the determinant of this matrix we again use the multilinear property, applying it to the row vectors in the second block (among three horizontal blocks separated by the single lines). After some algebraic manipulation, one has

$$
\operatorname{det} \boldsymbol{N}_{\nu}=\Phi_{\nu}^{r} r_{\varphi_{\nu}}^{\prime}(-\mu)^{k} \prod_{j=1}^{k}\left(\lambda_{j}-\mu\right)
$$

Finally, substitution of the derivatives computed in (G.5) allows to complete the computation started in (G.19). One has

$$
\operatorname{det}(\boldsymbol{L}-\lambda \boldsymbol{I})=(1-\mu)^{k-1}(-\mu)^{k-1}(\mu-1+\bar{e})\left(\mu \prod_{j=1}^{k}\left(\lambda_{j}-\mu\right)+\frac{1-\mu}{\left\langle x^{2}\right\rangle} \sum_{j=1}^{k}\left(\left(1-\lambda_{j}\right) f_{j, y}^{\prime} \varphi_{j}^{*} \prod_{i=1, i \neq j}^{k}\left(\lambda_{i}-\mu\right)\right)\right)
$$

Combining now (G.17), (G.18) and the last expression, we get polynomial (G.16).

Using the characteristic polynomial of the Jacobian matrix in the corresponding equilibrium, it is straightforward to derive the equilibrium stability conditions of Section 4.3 .

\section{The case of one survivor: Proof of Proposition 4.2}

If $k=1$, characteristic polynomial (G.6) reduces to

$$
(-1)^{N} \mu^{N-1}\left(\lambda_{1}-\mu\right) \prod_{j=2}^{N}\left(\lambda_{j}-\mu\right)^{2}\left(\mu\left(\lambda_{1}-\mu\right)+\frac{\left(1+r^{*}\right) \mu-1}{x_{1}^{*}\left(1-x_{1}^{*}\right)} \cdot\left(1-\lambda_{1}\right) \cdot f_{1, y}^{\prime}\right) \prod_{j=2}^{N}\left(\frac{1+x_{j}^{*}\left(r^{*}+\bar{e}\right)}{1+r^{*}}-\mu\right) \cdot
$$

Since $\lambda_{j} \in[0,1)$ there are $3 N-2$ roots that are inside the unit circle irrespective of the model parameters. The conditions in (4.12) are derived from the requirement

$$
\left|\frac{1+x_{j}^{*}\left(r^{*}+\bar{e}\right)}{1+r^{*}}\right|<1 \quad j \geq 2,
$$

which comes from the last factor. The three inequalities in (4.11) are obtained applying standard conditions for the roots of the second degree polynomial in the first parentheses, which is analogous to (D.1). The Proposition 4.2 is proved. 


\section{The case of many survivors: Proof of Proposition 4.3 and Corollary 4.2}

In the case of $k>1$ survivors the characteristic polynomial in (G.6) possesses a unit root with multiplicity $k-1$. Consequently, the fixed point is non-hyperbolic. To find the eigenspace associated with the eigenvalue 1 we subtract from the initial Jacobian matrix (G.1) computed at the equilibrium the identity matrix of the corresponding dimension and analyze the kernel of the resulting matrix $\boldsymbol{J}-\boldsymbol{I}$. This can be done through the analysis of the kernel of the matrix obtained by the substitution of the identity matrix from matrix $\boldsymbol{L}$ given in (G.9). Let us consider the $k<N$ and the $k=N$ cases separately.

When $k<N$, as we showed in the proof of Lemma G.2, in the resulting matrix the last $k-1$ columns are identical, see (G.10) and (G.11). Therefore, the kernel of the matrix $\boldsymbol{J}-\boldsymbol{I}$ can be generated by a basis containing the following $k-1$ vectors

$$
\boldsymbol{u}_{n}=(\underbrace{0, \ldots, 0}_{N} ; \underbrace{0, \ldots, 0}_{N} ; \underbrace{0, \ldots, 0}_{N} ; \underbrace{0, \ldots, 0}_{n-1}, 1, \underbrace{0, \ldots, 0}_{k-n-1},-1 ; \underbrace{0, \ldots, 0}_{N-1-k}), \quad 1 \leq n \leq k-1 \quad .
$$

Notice that the direction of vector $\boldsymbol{u}_{n}$ corresponds to a change in the relative wealths of the $n$-th and $k$-th survivor.

If, instead, $k=N$, then the last $k-1$ columns in the resulting matrix are zero vectors, and then the kernel of the matrix $\boldsymbol{J}-\boldsymbol{I}$ can be generated with the $N-1$ vectors of the canonical basis

$$
\boldsymbol{v}_{n}=(\underbrace{0, \ldots, 0}_{N} ; \underbrace{0, \ldots, 0}_{N} ; \underbrace{0, \ldots, 0}_{N} ; \underbrace{0, \ldots, 0}_{n-1}, 1, \underbrace{0, \ldots, 0}_{N-n-1}), \quad 1 \leq n \leq N-1
$$

whose direction corresponds to a change in the relative wealths of the $n$-th and $N$-th survivors.

If the system is perturbed away from equilibrium $\boldsymbol{x}^{*}$ along the directions defined in (G.20) or (G.21), a new fixed point is reached. Then, the system is stable, but not asymptotically stable, with respect to these perturbations.

Moreover, since the eigenspaces identified above do not depend on the system parameters, it is immediate to realize that they do constitute not only the tangent spaces to the corresponding non-hyperbolic manifolds, but the manifolds themselves.

The polynomial (4.13) is the last factor in (G.6), while conditions (4.14) are obtained by imposing

$$
\left|\frac{1+x_{j}^{*}\left(r^{*}+\bar{e}\right)}{1+r^{*}}\right|<1 \quad j>k+1,
$$

which completes the proof of Proposition.

If all the survivors are characterized by the same parameter $\lambda \in[0,1)$, the last factor in (G.6) reduces to

$$
(\lambda-\mu)^{k-1}\left(\mu(\lambda-\mu)+(1-\lambda) \frac{\left(1+r^{*}\right) \mu-1}{x_{1 \diamond k}^{*}\left(1-x_{1 \diamond k}^{*}\right)} \sum_{j=1}^{k} \varphi_{j}^{*} f_{j, y}^{\prime}\right)
$$

and the result of Corollary directly follows applying standard conditions to the second-degree polynomial in the parenthesis above.

\section{The case of "no-arbitrage" equilibria: Proof of Proposition 4.4 and Corollary 4.3}

Independently of the number of survivors, characteristic polynomial in (G.16) possesses a unit root with multiplicity $N-2$. Consequently, the fixed point $\boldsymbol{x}^{*}$ is never hyperbolic, when $N \geq 3$. It is easy to see that in this case all equilibria belong to the manifold of dimension $N-2$ and that this is exactly a non-hyperbolic manifold of $\boldsymbol{x}^{*}$. For the stability of equilibrium $x^{*}$ with respect to the perturbations in the directions orthogonal to this manifold, it is sufficient to have all other eigenvalues inside the unit circle. If this condition is satisfied, then equilibrium $\boldsymbol{x}^{*}$ of the system is stable, but not asymptotically stable. Since $\lambda_{j}<1$ for all $j$ and since $\bar{e}>0$, this sufficient condition can be expressed through the roots of the last term in (G.16). This term is exactly polynomial (4.16).

If all the survivors are characterized by the same parameter $\lambda \in[0,1)$, the last factor in (G.16) reduces to

$$
(\lambda-\mu)^{k-1}\left(\mu(\lambda-\mu)+(1-\lambda) \frac{1-\mu}{\left\langle x^{2}\right\rangle} \sum_{j=1}^{k} \varphi_{j}^{*} f_{j, y}^{\prime}\right)
$$

and the result of Corollary directly follows applying standard conditions to the second-degree polynomial in the parenthesis above. 


\section{References}

Amir, R., I.V. Evstigneev, T. Hens and K.R. Schenk-Hoppé, (2005). "Market Selection and Survival of Investment Strategies", Journal of Mathematical Economics, 41, n.1/2, 105-122.

Anufriev, M. and G. Bottazzi, (2005). "Price and Wealth Dynamics in a Speculative Market with an Arbitrary Number of Generic Technical Traders", L.E.M. Working Paper Series, 2005/06, Sant'Anna School for Advanced Studies, Pisa.

Arthur, W.B., J.H. Holland, B. LeBaron, R. Palmer, P. Tayler, (1997). “Asset Pricing under Endogenous Expectations in an Artificial Stock Market", in Arthur W.B., S.N. Durlauf and D.A. Lane (eds.) The Economy as an Evolving Complex System II, Addison-Wesley.

Barberis, N. and R.H. Thaler, (2003). "A Survey of Behavioral Finance”, in Constantinides G.M., M. Harris and R. Stultz (eds.) Handbook of the Economics of Finance, Elsevier Science, North Holland, Amsterdam.

Blume, L. and D. Easley, (1992). "Evolution and Market Behaviour", The Journal of Economic Theory, 58, 9-40.

Bottazzi, G. (2002). “A Simple Micro-Model of Market Dynamics. Part I: the "Large Market” Deterministic Limit” L.E.M. Working Paper, 2002-10, Sant'Anna School for Advanced Studies, Pisa.

Bottazzi, G., G. Dosi and I. Rebesco, (2005). "Institutional Achitectures and Behavioural Ecologies in the Dynamics of Financial Markets: a Preliminary Investigation", Journal of Mathematical Economics, 41, n.1/2, 197-228.

Brock, W.A. and C.H. Hommes, (1998). "Heterogeneous Beliefs and Routes to Chaos in a Simple Asset Pricing Model", Journal of Economic Dynamics and Control 22, 1235-1274

Brock, W.A., C.H. Hommes and F.O.O. Wagener, (2005). "Evolutionary Dynamics in Markets with Many Trader Types", Journal of Mathematical Economics, 41, n.1/2, 7-42.

Campbell, J.Y. and L.M.Viceira, (2002). Strategic Asset Allocation: Portfolio Choice for Long-Term Investors, Oxford University Press, Oxford.

Chiarella, C. (1992). "The Dynamics of Speculative Behaviour", Annals of Operations Research, 37, 101-123.

Chiarella, C., R. Dieci and L. Gardini, (2002). "Speculative behaviour and complex asset price dynamics: a global analysis", Journal of Economic Dynamics and Control 49, 173-197.

Chiarella, C. and X. He, (2001). "Asset Price and Wealth Dynamics under Heterogeneous Expectations", Quantitative Finance, 1, 509-526.

Chiarella, C. and T. He, (2002a). "Heterogeneous Beliefs, Risk and Learning in a Simple Asset Pricing Model", Computational Economics, 19 (1), 95-132.

Chiarella, C. and T. He, (2002b). "An Adaptive Model on Asset Pricing and Wealth Dynamics with Heterogeneous Trading Strategies", Quantitative Finance Research Centre, University of Technology, Sydney, Working Paper Number: 84. 
DeLong, J.B., A. Shleifer, L.H. Summers and R.J. Waldmann (1990). "Noise trader risk in financial markets", The Journal of Political Economy, 98, n.4, 703-738.

DeLong, J.B., A. Shleifer, L.H. Summers and R.J. Waldmann (1991). “The survival of noise traders in financial markets", The Journal of Business, 64, n.1, 1-19.

Farmer, J.D. (2002). "Market force, ecology, and evolution", Industrial and Corporate Change, 11, 895-953.

Gaunersdorfer, A. (2000). "Endogenous Fluctuations in a Simple Asset Pricing Model with Heterogeneous Agents", Journal of Economic Dynamics and Control, 24, 799-831

Hens, T. and K.R.Schenk-Hoppé, (2005). "Evolutionary Stability of Portfolio Rules in Incomplete Markets", Journal of Mathematical Economics, 41, n.1/2, 43-66.

Hommes, C.H. (2005) "Heterogeneous agents models in economics and finance". In: Judd K., L. Tesfatsion (eds) "Handbook of Computational Economics II: Agent-Based Computational Economics". Elsevier, North-Holland (forthcoming).

J.P.Morgan (1996). RiskMetrics ${ }^{T M}$ - Technical Document, 4th ed., Morgan Guaranty Trust Company of New York, New York.

Kirman, A. (1993). "Ants, rationality and recruitment", Quarterly Journal of Economics, 108, 137156.

LeBaron B., W.B. Arthur and R. Palmer (1999). "Time series properties of an artificial stock market", Journal of Economic Dynamics and Control, 23, 1487-1516.

LeBaron B. (2005). “Agent-based Computational Finance”. In: Judd K., L. Tesfatsion (eds) "Handbook of Computational Economics II: Agent-Based Computational Economics". Elsevier, NorthHolland (forthcoming).

Levy M., H. Levy and S. Solomon (1994). "A Microscopic Model of the Stock Market: Cycles, Booms, and Crashes", Economics Letters 45, 1, 103-111.

Levy M., H. Levy and S. Solomon (1995). "Microscopic simulation of the stock market: the effect of microscopic diversity", Journal of Physica I (France) 5, 1087-1107.

Levy M., H. Levy and S. Solomon, (2000). Microscopic Simulation of Financial Markets, Academic Press, London.

Lux T., (1995). "Herd Behaviour, Bubbles and Crashes", The Economic Journal, 105, no. 431, 881896.

Lux, T. and M. Marchesi, (1999). "Scaling and criticality in a stochastic multi-agent model of financial market", Nature, vol. 397, 498-500.

Samuelson P., (1969). "Lifetime Portfolio Selection by Dynamic Stochastic Programming", Review of Economics and Statistics, 51, 239-246.

Zschischang, E. and T. Lux (2001). "Some new results on the Levy, Levy and Solomon microscopic stock market model”, Physica A, 291, 563-573. 